Provided for non-commercial research and educational use only. Not for reproduction or distribution or commercial use.

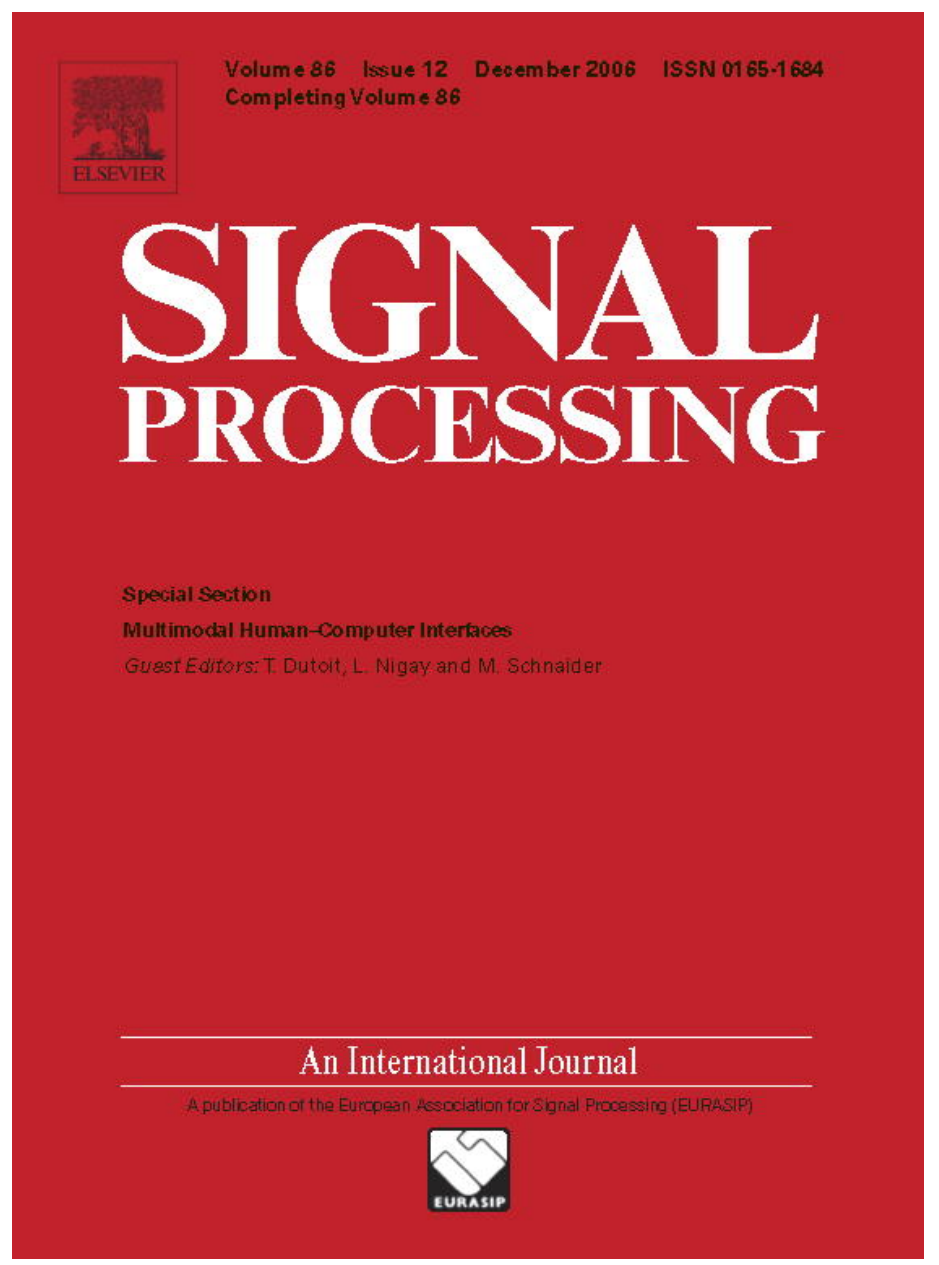

This article was originally published in a journal published by Elsevier, and the attached copy is provided by Elsevier for the author's benefit and for the benefit of the author's institution, for non-commercial research and educational use including without limitation use in instruction at your institution, sending it to specific colleagues that you know, and providing a copy to your institution's administrator.

All other uses, reproduction and distribution, including without limitation commercial reprints, selling or licensing copies or access,

or posting on open internet sites, your personal or institution's website or repository, are prohibited. For exceptions, permission may be sought for such use through Elsevier's permissions site at: 


\title{
Sensory substitution using tactile pin arrays: Human factors, technology and applications
}

\author{
Steven A. Wall*, Stephen Brewster \\ Glasgow Interactive Systems Group, Department of Computing Science, University of Glasgow, 17 Lilybank Gardens, G12 8QQ, UK
}

Received 1 July 2005; received in revised form 5 December 2005; accepted 1 February 2006

Available online 8 June 2006

\begin{abstract}
Tactile arrays use a matrix of individually controllable elements to present spatial and temporal patterns of cutaneous information. Early devices of this type were in the field of sensory substitution to replace vision or hearing for users with a sensory impairment. Many advances have been made due to the appropriation of tactile displays for telerobotics and virtual reality, to represent physical contact with a remote or simulated environment. However, many of these have been limited to engineering prototypes. The recent commercial availability of affordable, portable tactile pin arrays has provided renewed impetus to apply the technology to sensory substitution applications. Lack of access to digitally stored data can prove a significant barrier to blind people seeking careers in numerate disciplines. Tactile displays could potentially provide a discrete and portable means of accessing graphical information in an intuitive non-visual manner. Results are presented from experiments on tactual perception related to understanding graphs and simple visualisations with a commercially available tactile array device. It was found that subjects could discriminate positive or negative line gradient to within $\pm 4.7^{\circ}$ of the horizontal, compared to $\pm 3.25^{\circ}$ for results with a force feedback mouse and $\pm 2.42^{\circ}$ with a raised paper representation.
\end{abstract}

(C) 2006 Elsevier B.V. All rights reserved.

Keywords: Tactile; Blind; Haptic; Multimodal

\section{Introduction}

The area of haptic (touch-based) human-computer interaction has grown rapidly over the last few years. A range of new applications has become possible now that touch can be used as an interaction technique. Most research in this area has been concentrated on the use of force feedback devices for the simulation of contact forces when interacting with simulated objects. Devices such as

\footnotetext{
*Corresponding author. Tel.: + 441413308430 .

E-mail addresses: steven@dcs.gla.ac.uk (S.A. Wall), stephen@dcs.gla.ac.uk (S. Brewster).
}

the Phantom haptic interface (see Fig. 1) from Sensable Technologies [1] present the illusion of contact with rigid virtual objects using programmable constraint forces supplied to an end effector such as a thimble, handle or stylus that the user interacts with. A typical device will take the form of a mechanical framework capable of movement along one or more axes (typically two or three for most applications). Movement of the end effector within this space is tracked by the device; this position is then compared to the position of virtual objects within the space. Collisions with these objects are rendered by using actuators (which can be pneumatic, electromechanical, or using a braking 


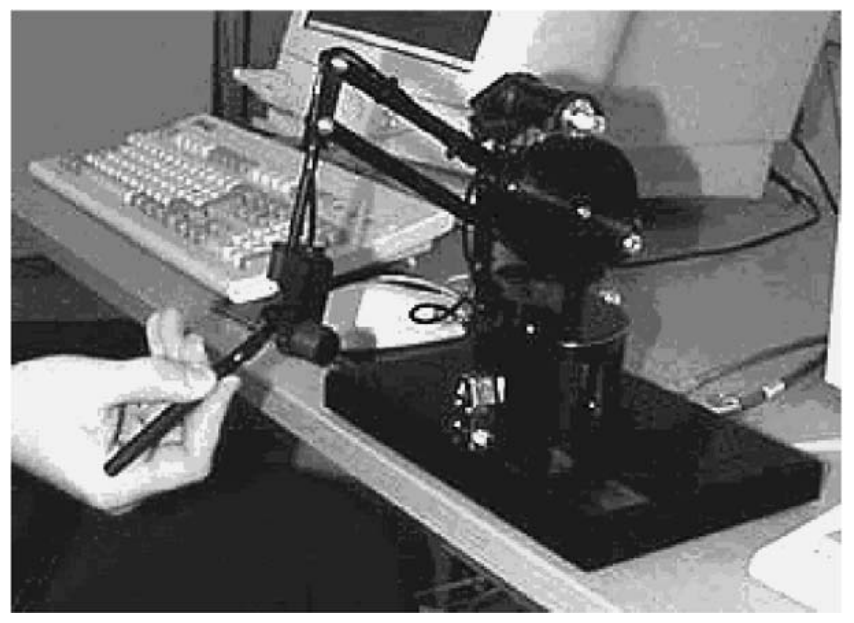

Fig. 1. The Phantom haptic interface. The user grips the stylus to interact with the device. Motion is selectively constrained to convey the illusion of contact with simulated objects.

system) to constrain the motion of the user to the surfaces of simulated objects.

Broadly speaking, the human haptic sense can be divided in to two distinct channels of sensory experience. Kinaesthetic information refers to the sensation of positions, velocities, forces and constraints that arises from the muscle spindles and tendons. Force feedback haptic interfaces appeal to the kinaesthetic senses by presenting computer controlled resistive forces to create the illusion of contact with a rigid surface. The cutaneous class of sensations refers to those which arise through direct contact with the skin surface. Cutaneous stimulation can be further subdivided in to the sensations of pressure, stretch, vibration, and heat. In some instances pain is also referred to as a separate sensation, though excessive stimulation of the other detectable parameters will lead to a feeling of pain.

Exploring a virtual world through a pen-like stylus or a thimble currently gives little provision for cutaneous sensations in force feedback devices, such as the complex distribution of forces on the skin that are perceived when placing a fingertip on a textured surface. Tactile display research seeks to replicate these sensations by using an array of individually controllable mechanical elements to perturb the skin at the user's fingertip (see Fig. 2). Providing distributed cues for surface qualities greatly expands the scope for potential applications of haptic displays [2]. For example, representing the very fine and subtly varying cues for tactile assessment of fabric is problematic by force feedback alone. Tactile displays could allow shoppers

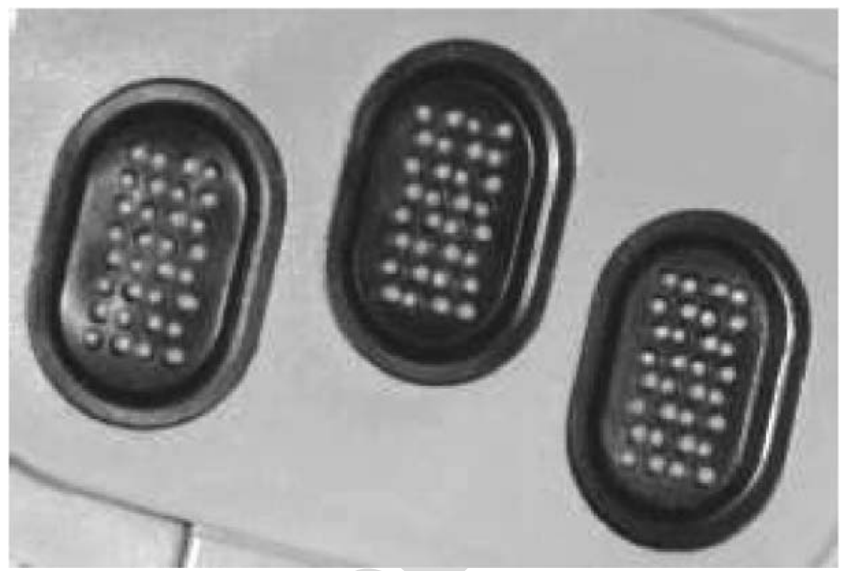

Fig. 2. A tactile pin array for fingertip display, consisting three, 4 $\times 8$ arrays of pins.

and fashion designers to experience clothing materials via the Internet to aid with online purchasing decisions $[3,4]$. Tactile display could also provide increased realism in medical training simulations, allowing the user to differentiate between different types of tissue more easily. This is particularly salient for applications that require direct palpation with the fingertips, rather than being mediated via surgical equipment, such as the diagnosis of bovine pregnancy [5].

As early as the 1920s, researchers were interested in using vibration of the skin as a means of information transfer (for example, Gault in 1926, cited in [7]). Tactile-vision substitution systems (TVSS) were the earliest to be developed, in order to present visual information to blind people. In a typical system, a camera receives visual information which is converted to a tactile representation on a two-dimensional pin array. Some of the earliest works in this area were the development of the Optacon (see Fig. 3), which converted printed letters to a spatially distributed vibrotactile representation on the fingertip, using a miniature handheld camera (summarised in [8]). Although reading speeds were significantly slower than Braille, the Optacon allowed blind people to access any text or graphics without having to wait for it to be converted into Braille. Early pioneering work in TVSS was also performed by Paul Bach-y-rita and colleagues in the late 1960s. Early systems displayed visual information captured by a tripod mounted TV camera to a vibrotactile display on the user's back. Due to limited spatial resolution, tactile masking effects and a low dynamic range, the system was not suitable as a day-to-day navigation aid. However, 


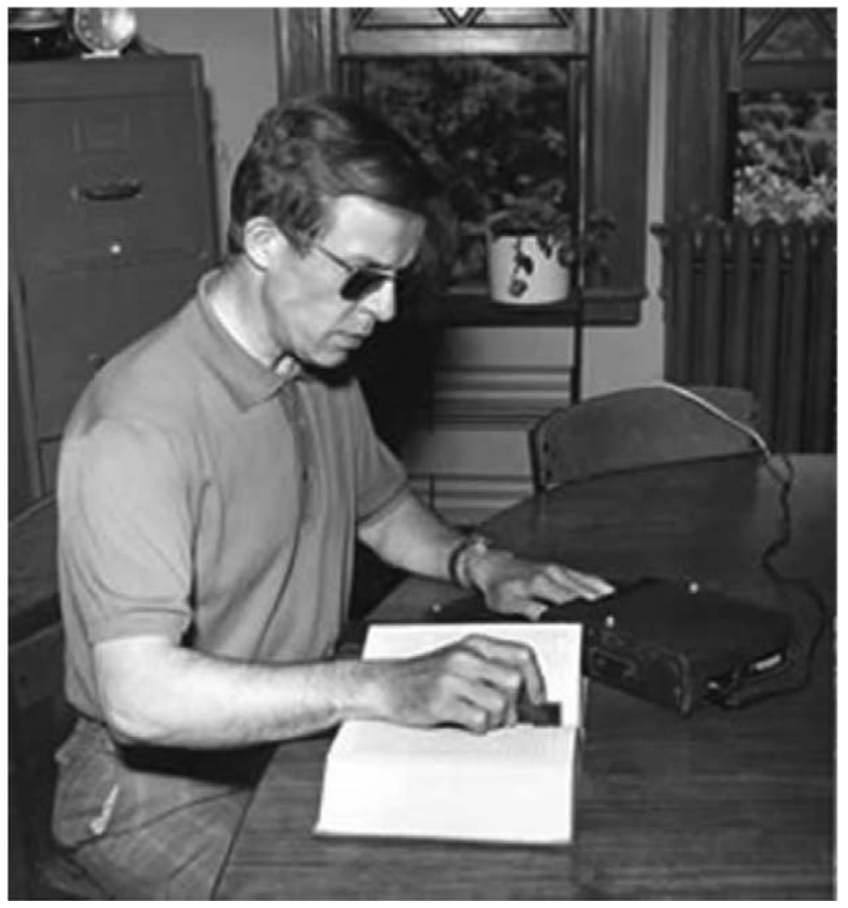

Fig. 3. Visually impaired person using the Optacon device to read text. The handheld camera detects light and dark areas of the page and converts to a vibrotactile representation, presented to the non-dominant hand on the pin array (Image used with permission from www.carroll.org).

subjects could easily recognise simple shapes and discriminate orientation of lines. It was also reported that experienced users could perform more complex tasks, such as recognition of faces, or electronic assembly using the system (reported in [9]).

Increases in computing power, the notions of "virtual reality", and the commercial availability of haptic force feedback devices as a research tool have effectively shifted the main emphasis of tactile display research from representing remotely sensed real-world information to the challenges inherent in interacting with an environment consisting of simulated physical models on a computer. The concept of connecting haptic feedback to a virtual world was first voiced in 1965 by Ivan Sutherland, who put forward a vision for an "ultimate display". Inspired by Sutherland's vision, Frederick Brooks jr. and his colleagues became the first team to realise force feedback with a graphical environment in the GROPE project (for an excellent treatise of the development of haptic feedback displays the authors recommend [10]). The scope of force feedback enabled applications expanded considerably with the development of the Phantom, the first commercially available desktop force feedback device [1]. The drive to create realistic cutaneous stimulation for virtual environments could now be said to be the motivation behind most tactile display research. In particular, the need to combine tactile displays with force feedback displays has led to increased efforts to miniaturise the technology.

Researchers have sought to appropriate haptic feedback devices for presenting information to visually impaired computer users. For example, Sjostrom outlined a number of guidelines for using haptic technology to provide novel computer interaction techniques for visually impaired people [11]. These include "rules of thumb" for navigation, gaining an overview, understanding objects, haptic widgets and physical interaction. Several projects have focussed on presenting simple visualisation such as graphs, charts and tables using haptic feedback technology. Graphs provide a means by which sighted people can access numerical information in a manner which affords quick, visual identification of trends, maxima, minima, intersection points, and other features that would be laborious and time consuming with a table of numerical data. Fritz and Barner reported the earliest work that used a Phantom to present graphs to visually impaired users [12]. Wies et al. reported work using the Logitech Wingman force feedback mouse to present graphical information [13]. The most extensive body of work in this area was performed by $\mathrm{Yu}$, Brewster and colleagues on the Multivis project (www.multivis.org). This project adopted a multimodal approach to presenting visualisations using force feedback and stereo sound. In a series of experiments, multimodal feedback was found to offer significant advantages over a single modality alone [14-16].

Due to their more compact size and power requirements, tactile displays offer a potentially much more discrete, affordable means of providing access to data visualisations via the sense of touch. Displays are often small enough to allow mounting on another, standard human-computer interaction device such as a mouse, keyboard or games controller, or portable devices such as mobile phones and personal digital assistants (PDAs). This paper reviews the developments in tactile displays for sensory substitution, along with relevant literature on perceptual and psychophysical issues related to the sense of touch. An experiment is described investigating perceptual issues related to graph exploration with a commercially available tactile display device. 


\section{Methods of tactile presentation for sensory substitution}

The earliest structured work in sensory substitution dates back to the 19th century, with the development of embossed raised characters for visually impaired people. Many "low-tech." solutions are still employed for presenting visualisations of numerical data, although they are being gradually augmented by microprocessor-based technology. In recent years several technologies have emerged which can be used to present information to the sense of touch, including vibration (vibrotactile), force feedback and tactile displays.

\subsection{Raised static material display}

Although several competing alphabets of embossed characters were developed around 1840, Louis Braille was the first to demonstrate the efficiency of raised dot patterns over simplifications of print letters. Each Braille character consists of a 3 row $\times 2$ column "cell", within which it is possible to form 64 individual patterns. Meanings assigned to these patterns include the letters of the alphabet, punctuation marks, and various contractions that stand for frequently recurring letter groups. Despite opposition from education on the grounds that sighted teachers and visually impaired students would require different representations, and the supposedly arbitrary nature of the encoding in dot patterns, practical considerations led to its widespread acceptance within the blind and visually impaired communities. Inspired by the seminal work of William Wait in the late 19th century (cited in [17]), experimental evidence was gradually accrued demonstrating the increase in efficacy observed with the Braille code over embossed letters in terms of reading speed and comprehension of text. The main drawback of Braille is that reading speeds are considerably slower than for visually presented text, around 104 words per minute (wpm) for experienced adult users [17] (in comparison, the average reading speed for sighted high school students is $250-300 \mathrm{wpm}$, with some adults reaching two or three times that speed [18]).

Raised paper diagrams, produced via embossing or heat-raised paper, have been employed for presentation of pictorial information including illustrations, photographs, graphs, and maps to the visually impaired. Petrie [19] and Challis [20] both describe the use of tactile embossed diagrams as an inexpensive computer interface for visually impaired users. Embossed diagrams still possess many advantages over more hi-tech solutions even today - they are cheap to produce, have no moving parts, and can be quickly and easily explored with the whole of both hands to provide a good overview of the information being displayed. They are, however, limited in size, can become easily cluttered with information, are subject to wear and tear and are inherently non-dynamic in nature. The "Talking Tactile Tablet" (T3), developed in conjunction with the Royal National College for the Blind in Hereford, UK, exploits the benefit of the tactile embossed format by combining a touch-sensitive tablet with traditional tactile diagrams and digitally stored audio information to provide dynamic, nested information for the visually impaired, beyond what is possible with a sheet of paper. For example, the system allows visually impaired access to the contents of a world encyclopaedia through a raised paper tactile map [21].

\subsection{Vibrotactile}

Vibrotactile displays consist of a single element stimulator that is used to encode information in temporal parameters of the vibration signal. Parameters that can potentially be employed include frequency, amplitude, waveform, duration and rhythm [22]. Stimulation can be generated by various means including, but not limited to, solenoids, voice coils or rotation of an inertia by a motor. Most people make use of vibrotactile technology within mobile phones, as a non-audiobased indicator of an incoming call or text message. Vibrotactile information has also found widespread acceptance within the video gaming community as an inexpensive means of providing touch feedback in hand held controllers. Actuators can produce tactile sensations used to represent impacts, firing of weapons or environmental effects. A small number of gaming software applications have employed vibrotactile display for abstract encoding of information, such as warning indicators, progress bars or tactile "radars". Researchers are currently investigating how more sophisticated vibrotactile parameters can be used to represent information [23], and how vibrotactile feedback can be combined with other sensory modalities and novel interaction methods to create compelling interactions for hand held devices [24]. 


\subsection{Force Feedback}

The need to represent textures and other high spatial frequency details of haptic virtual objects has led many researchers to investigate the presentation of tactile information using force feedback devices. The most common method of rendering haptic sensations with force feedback devices is the point interaction model, whereby the user is represented in the virtual environment by a single point corresponding to the tip of a probe or thimble through which the user interacts with the device. This situation is analogous to exploring objects remotely through an intermediary link, such as a stick or probe. In this situation, the distributed spatial cues on the fingertip correspond to the geometry of the probe, rather than the surface of the object being explored. However, it has been shown that it is still possible to estimate the surface qualities of a texture explored via vibrations arising through dynamic contact between the probe and the texture [25]. This is an example of the phenomenon of "distal attribution", whereby the resulting vibration sensations are attributed to the properties of the surface at the distal point of the probe, rather than as arising directly from the probe itself [26].

The earliest reported work in the area of force feedback representations of texture was the sandpaper system, which represented texture as a series of lateral forces created by "virtual springs", rendered on a force feedback joystick [27]. Massie describes representation of textures on a Phantom haptic interface using sinusoidal perturbations perpendicular to the object surface [28]. Several methods of texture rendering have been investigated, including stochastic [29], image-based and fractal [30], and Fourier series [31] models. However, it is perhaps fair to say that perception of texture via force feedback devices is not fully understood. In particular, robust models of the relationship between the simulated physical characteristics of the texture and user perception remain elusive at present. A greater understanding of how force feedback devices mediate human perception also needs to be developed, for example, results on the range of a perceptually stable texture space have already been investigated by researchers at Purdue University [32].

\subsection{Tactile displays}

Tactile displays can be divided roughly in to three categories of stimulation: electro-cutaneous, ther- mal, and mechanical. Electro-cutaneous displays create touch sensations by passing a small electric current through the skin. They are advantageous as they contain no moving parts, therefore construction, control and maintenance is relatively simple. They are more limited in application than mechanical displays due to the relatively small difference in the thresholds between signal detection and the onset of pain. The reported range of stimulation varies between 6 and $20 \mathrm{~dB}$; for comparison purposes the vibrotactile range of skin is around $40 \mathrm{~dB}[9,33]$. Thermal displays have generated some research interest, but are yet to find sufficient purchase in commercial devices due to the lack of an actuator of a suitable bandwidth, safety issues and the fact that human thermal sensing is relatively little understood compared to the other two modes of stimulation [34,35].

Mechanical tactile displays utilise actuated components to actively deform the user's skin via pressure, stretch or other means, in order to induce programmed touch sensations. They can be further categorised by their method of stimulation in to vibration, lateral displacement (skin stretch) and skin indentation. Vibration displays present shape information via activating patterns of spatially configured transducers at high temporal frequencies of operation. These elements tend to be much smaller than the vibrotactile transducers described in Section 2.2, for example, pins or wires driven by solenoids or piezoelectric actuators [36]. Devices for lateral displacement present information through spatiotemporal patterns of skin stretch [37,38]. Tactile displays for skin indentation present distributed cues by inducing pressure on the skin via a number of moving elements. They have received the most attention for virtual environment applications as they offer the most significant potential to represent the fingertip deformations that occur in touch interaction with everyday objects. There is also some evidence that a static tactile display is superior to a vibrating display. Embossed letters are recognised tactually with the finger pad at 50\% accuracy if their height is 4-6 mm; similar accuracy with the Optacon requires $12-20 \mathrm{~mm}$ height $[39,40]$.

\section{Human tactile perception}

The physiological basis of haptic perception carries profound implications for the design of tactile display devices. Similarly, many relevant psychophysical studies have been conducted, which 
may indicate the degree of information that can be transmitted by mechanical perturbation of the skin. Here we discuss factors arising from neurophysiological, psychophysical and perceptual studies of the sense of touch.

\subsection{Mechanoreceptors of the glabrous skin}

There are four types of low threshold, mechanoreceptive nerve endings in the hairless (glabrous) skin of the human hand. They can be classified based upon the size of their receptive field, and their response to a step indentation of the skin. A mechanoreceptor's response is measured by the firing rate on it's corresponding afferent nerve fibre. Rapidly adapting (RA) units have little to no static response, discharging only as long as the skin stimulus is in motion. RA units can be further classified in to Type I (small receptive field) and Type II (large receptive field). Slowly adapting (SA) receptor types are less sensitive to dynamic stimuli than the RA units, but exhibit a response dependant on the amplitude of maintained skin indentation. They can also be classified in to Type I (small) and Type II (large) variants depending on the size of their receptive field. For all types of mechanoreceptor, the frequency range within which the units respond is increased rapidly with the amplitude of the stimulus [41,42]. The RAII receptors are also frequently referred to as Pacinian corpuscles (PC) in the literature. The characteristics of the four main types of mechanoreceptor are summarised in Table 1.

Experimental correlates between psychophysical studies and neurophysiologic measures suggests the mechanoreceptive afferents can also be categorised by function [43]. SAI receptors are responsible for the detection of form and texture of objects; they have a high sensitivity to temporal and spatial structure of stimuli. Comparatively, SAII afferents are less sensitive to skin indentation, but more sensitive to lateral skin stretch and are therefore implicated in the perception of relative movement against a surface. RAI mechanoreceptors are more sensitive than the SAI receptors, but have a poorer spatial resolution. As a result of this they present a good indication of motion, and are therefore useful in tasks such as slip detection and grasping, but less so in tasks that require spatial acuity. The RAII system is extremely sensitive to high-frequency vibrations. Perception of vibration is of great importance when perceiving remote contact via an intermediary link, such as a tool, probe or a haptic force feedback device. Because of their deeper location in the skin they have essentially no spatial acuity in comparison to the other receptor systems.

The "duplex model" of touch postulates two channels of stimulation, PC (corresponding to the Pacinian corpuscles, the RAII receptors) and nonPC (encompassing the other mechanoreceptive afferents in the glabrous skin). The two channels are distinguished by their spatial and temporal response to stimulations. For frequencies lower than $40 \mathrm{~Hz}$, thus primarily exciting the non-PC channel, it was shown that sensation threshold is largely independent of contact area. For frequencies of stimulation greater than $40 \mathrm{~Hz}$, therefore appealing to the PC channel, the effect of contact area has been illustrated to be one of the most salient factors in terms of determination of indentation thresholds. Sensitivity increased with contactor area, illustrating that spatial summation of signals occurs for higher frequencies [44].

Results with a vibrotactile array showed a greater ability to resolve signals within noise at higher frequencies designed to stimulate the $\mathrm{PC}$ receptors than at lower frequencies intended to stimulate the non-PC population. Detection rates were also markedly better when the background masking stimulus was targeted at the alternate receptor group (e.g. detecting a high-frequency moving vibratory signal in low-frequency background noise

Table 1

Properties of the mechanoreceptive afferents of the glabrous skin of the human hand [33, 41, 43]

\begin{tabular}{lllll}
\hline Receptor type & $\begin{array}{l}\text { Receptive field area } \\
\left(\mathrm{mm}^{2}\right)\end{array}$ & $\begin{array}{l}\text { Frequency range (peak } \\
\text { sensitivity) }(\mathrm{Hz})\end{array}$ & $\begin{array}{l}\text { Amplitude threshold for } \\
\text { vibration }(\mu \mathrm{m})\end{array}$ & Effective stimulus \\
\hline RAI & $1-100$ & $1-300(50)$ & 2 & Skin motion \\
SAI & $2-100$ & $0-100(5)$ & 30 & Edges, points, corners \\
RAII (PC) & $10-1000$ & $5-1000(250)$ & 0.01 & Vibration \\
SAII & $10-500$ & $<8(0.5)$ & 40 & Skin stretch \\
\hline
\end{tabular}


and vice versa) [36]. Rogers [45] also noted an increased acuity for identification of tactile letters at higher frequencies. These results, however, cannot be solely attributed to the spatial acuity of the PC system, as vibrations at this frequency will also likely elicit a response from non-PC receptors $[36,46]$. Sherrick et al. also noted that spatial localisation performance appeared to be better for frequencies exciting the non-PC receptors $(25 \mathrm{~Hz}$ stimulus) than the $\mathrm{PC}$ system $(250 \mathrm{~Hz}$ stimulus) at various skin locations, but noting that performance would improve for the PC system at the fingertip due to the marked increase in density of receptors [47].

\subsection{Discrimination: absolute and difference thresholds}

The two points of contact discrimination threshold for the fingertips is widely regarded as $0.9 \mathrm{~mm}$ when the stimuli are placed against the subject's finger in the absence of any movement lateral to the skin's surface. Two points of contact closer than this threshold cannot be resolved in to distinct stimuli. Experimental evidence suggests that active exploration marginally increases sensitivity, decreasing the threshold to $0.7 \mathrm{~mm}$ (findings are summarised in [48]).

The most sensitive part of the human hand in terms of psychophysical thresholds for indentation is the fingertip, estimated by psychophysical studies to be in the region of $10 \mu \mathrm{m}$ for step indentations $[33,49]$. The highest thresholds for detection occur at low frequencies of stimulation [50]. Minimum thresholds occur in the region of $250 \mathrm{~Hz}$ stimulation $[44,45,50]$ corresponding to the maximum sensitivity of the RAII receptors [41]. Discrimination capacity for amplitudes is largely dependant on the location of stimulation, being finer at those points of the body with lower detection thresholds [51]. As with the vision and auditory senses, discrimination capacity is not constant throughout the stimulation intensity scale. Indentation amplitude discrimination is lower resolution at low intensities, becomes more sensitive in the range $200-700 \mu \mathrm{m}$, progressively degrading with increasing stimulus intensity [52]. Discrimination capacity for amplitudes is reported to be roughly independent of frequency of stimulation [53].

The resolution of temporal frequency discrimination is finer at lower frequencies. Investigations by Goff involving the stimulation of the subject's finger with a single probe showed that for lower frequencies $(<25 \mathrm{~Hz})$, discrimination threshold was less than $5 \mathrm{~Hz}$. For frequencies greater than $320 \mathrm{~Hz}$, discrimination capacities were degraded [54]. Measures for discrimination thresholds of frequency are problematic, as perception of vibratory pitch is dependant not just on frequency, but also an amplitude of stimulation. This is similar to the effect of volume on pitch perception in audio, but the effect is more pronounced for vibratory stimuli on the skin. Geldard [55], as reported by Tan [53], found that subjects reported a change in pitch when frequency was fixed, but amplitude of stimulation was changed.

\section{Sensory substitution}

The process of sensory substitution involves the sensing of stimuli by electronic means, transformation of the stimulus via signal processing, and presentation of the transformed stimulus in another modality. The main application of these systems is increasing accessibility for those with sensory impairments. The earliest sensory substitution devices converted visual stimuli to tactile representations for the blind and visually impaired. There are also examples of visual-to-auditory [56] and auditory-to-tactile (see Section 4.3) substitution systems.

\subsection{The Optacon}

The Optacon was one of the first devices to employ a matrix of pins for tactile-vision substitution and was the first device of this kind to be developed as a commercial product. The input to the device is a $6 \times 24$ array of photosensitive cells, which detects patterns of light and dark as material is moved underneath. The display part of the device is a $6 \times 24$ array of pins on which the user places their fingertip. The output of the camera is represented by vibrating pins on the tactile display. The pins vibrate at a frequency of $230 \mathrm{~Hz}$, which is close to the maximum sensitivity of the $\mathrm{PC}$ receptors for vibration [44]. Users are encouraged to select vibration amplitude that maximises their comfort.

Reading speeds with the Optacon are around $10-12 \mathrm{wpm}$ after the initial 9 day training period, reaching $30-50 \mathrm{wpm}$ after further training and experience [8]. Hill extended the width of the Optacon display to allow simultaneous presentation of more than one letter. Visual reading rates 
increase with the number of letters displayed at any particular time, however, this was not the case for tactile reading ([57] cited in [8]). When only one or two columns of the display were activated, hence giving only a partial view of letters scanned with the camera, reading rates were found to decrease ([58] cited in [8]). Loomis et al found a similar result when tactual line drawings were presented: no significant improvement in recognition rate was found for a two-finger aperture, compared to one finger exploration [59]. Conversely, in a study using a TVSS that presented letters tactually to the user's back, it was found that narrowing the width of the display to only show a smaller portion of the letters actually improved recognition rates. It was proffered that the difference in results between this and the Optacon study may have been due to the greater amount of practice the Optacon subjects had with the display ([60] cited in [8]).

Bliss and colleagues found that a pattern moving across the display produced better recognition rates than a static presentation, in which all elements are turned on and off simultaneously ([61] cited in [8]). Conversely, in a later study a static mode of presentation was found to produce superior recognition rates for letters than a scan mode. For a display time of $26 \mathrm{~ms}$, the rate of correct letter recognition in static mode was $65 \%$, compared to approximately $25 \%$ in scan mode. The difference in performance decreased as the display time increased. At longer durations both modes had relatively similar levels of performance.

In an extensive series of investigations with the Optacon, it was found that backwards masking of stimuli (a second stimulus, or "masker", is presented after the stimulus to be identified) was more disruptive to performance in pattern recognition tasks than forward masking (masker is presented before the stimulus to be identified) [7]. Both static presentation of letters and scanning them across the fingertip showed similar masking effects (described in [8]). Increasing the time between onsets of successive stimuli is the critical factor in reducing the effect of masking, as opposed to increasing the inter-stimulus interval during which no information is presented. Therefore, simply making presentation of patterns briefer (hence increasing the gap between successive patterns) will not diminish the masking effect [7]. If the time interval between the stimuli and the application of the mask is greater than approximately $200 \mathrm{~ms}$, there is little interference from the masker (results described in [8]).
Spatially separating patterns presented to a single finger will reduce the effect of masking, particularly for short durations of onset between successive stimuli (results by Weisenberger cited in [7]). Increasing the amplitude of vibration has been shown to improve the recognition rate for individual letters, however, reading performance as a whole is largely independent of amplitude; this may be so as the increase in intensity of a single letter will also increase its effectiveness as a masking stimulus for adjacently presented letters [8].

Kops and Gardner investigated the discrimination of "textures" formed by periodic spacing of active pins on the Optacon. The textures varied in angular orientation (e.g. horizontal, vertical or oblique lines of elements) and their density (number of Optacon elements active). Texture patterns were scanned back and forth underneath the subjects' finger as it rested on the Optacon, with an exploration velocity equivalent to $60 \mathrm{~mm} / \mathrm{s}$. Textures that differed in both spatial orientation and density were the most easily discriminated. Stimulation intensity appeared to be the most salient factor for patterns that had a large difference in density; spatial orientation became more salient for discrimination of texture patterns with smaller densities. Low-density patterns were more accurately discriminated than high-density textures, and the horizontal and vertical orientations were more easily discriminated than the oblique patterns. The vertical texture was the only distinct pattern at high densities. There was also a strong correlation between the subjects' ability to match the tactile patterns with visual representations, and their ability to distinguish them tactually. In a less formal study with a sub-group of participants, discrimination ability was found to be significantly higher at slower exploration speeds $(30 \mathrm{~mm} / \mathrm{s})$ [62].

\subsection{Virtual tactile displays}

In order to allow exploration of a larger tactile image with a device limited to the size of one or two fingertips, several devices have adopted a strategy of mounting a tactile display on a computer input device, such as a mouse or graphics tablet stylus. This allows the motion of the user's fingertips to be tracked within the limits of a certain workspace and the tactile display to be updated accordingly, dependant on where the user is on the "virtual image". Kaczmarek and Bach-y-rita classify devices of this type as "virtual tactile tablets" [9], after the 
work of Boyd et al. [63]. We propose a more generic term, "virtual tactile display", which does not exclude devices that do not employ a tablet interface for input or output. They can be distinguished from devices such as the Optacon in that control and stimulation occur at the same body location, and they are most commonly employed to represent information that is stored digitally on a computer, rather than present in the user's distal environment. They can also be distinguished by the fact that "active" exploration is necessary in order to perceive the entirety of the image being displayed on the device. It is necessary for the user to integrate the device in to a proprioceptive-tactile perceptual feedback loop in order to synthesise a spatial mental representation of the stimulus from the temporally varying tactile cues at the fingertip and proprioceptive information regarding the position of the fingers (Fig. 4).

Early exponents of active exploration were Katz [64] and Gibson [65] who both recognised the importance of relative movement between skin and surface for tactile perception. Broadly speaking, active perception deals with the voluntary movement of skin over a surface. In a passive situation the stimulus is presented to the skin by the experimenter, this can be static presentation without movement, movement of the stimulus relative to the skin, or movement of the subject that is not induced voluntarily, but by the experimenter in some fashion, such as an actuated mechanical system. Initial allusions were that the notions of active and passive touch were mutually exclusive concepts. Lederman and Taylor [66], however, stated that active/passive did not represent a dichotomy, more that a method of exploration could have degrees of activity or passivity depending on the restriction of feedback channels to the subject. Kaczmarek provides a summary of this continuum within the context of tactile display research [9]. Jansson points out that the argument for active versus passive perception can also be seen as an argument for the relative importance of kinaesthetic and cutaneous information [67]. Indeed, active exploration would seem to be important for resolving tactile information larger than the fingertip. Jansson describes a study using an Optacon display mounted on a two degrees of freedom chassis capable of planar movements. The display's output was determined by a controlling PC, thus converting the device to a

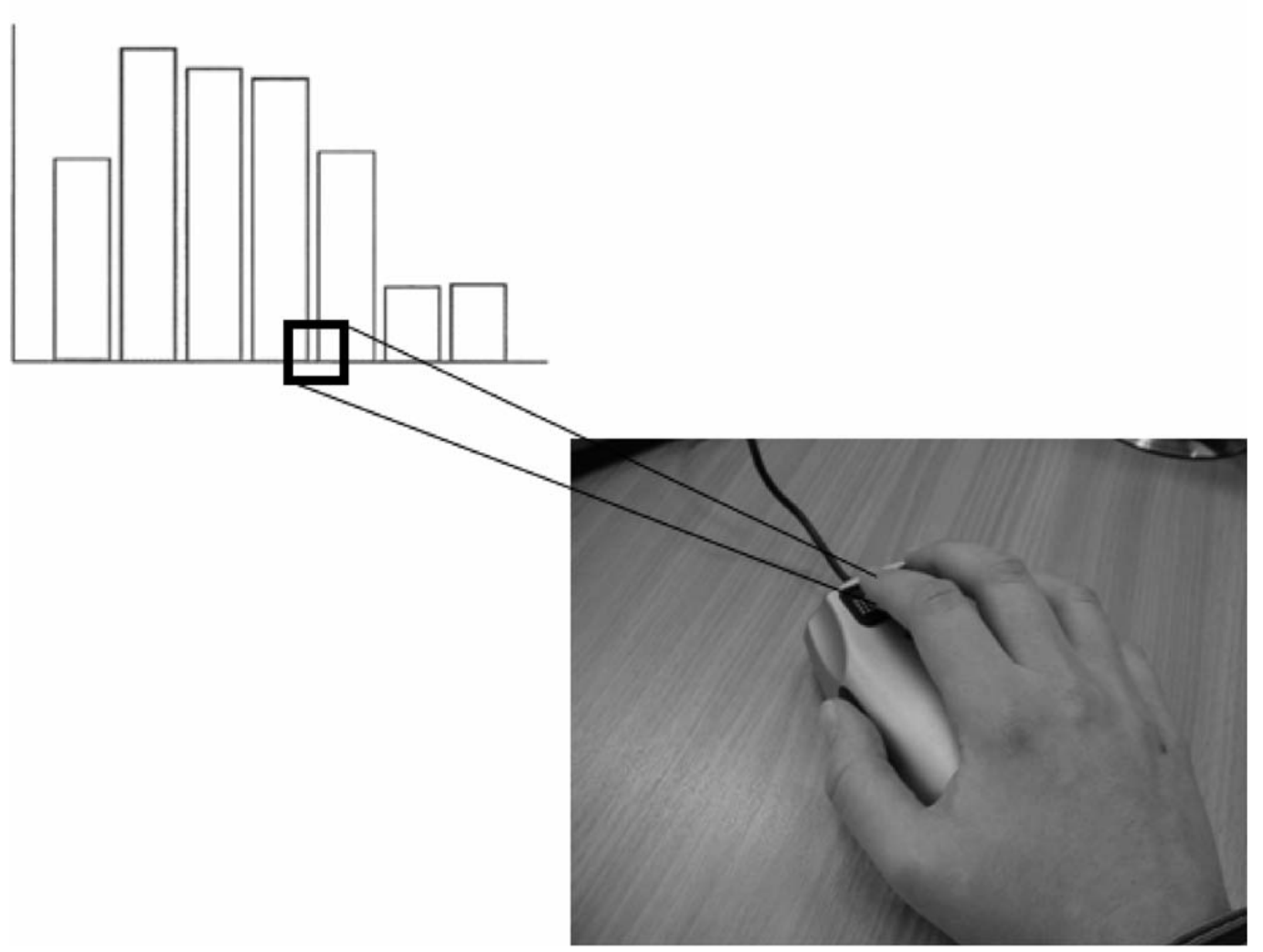

Fig. 4. User explores complete image with virtual tactile display but must construct a spatial image from tactile and proprioceptive cues over time. 
virtual tactile display. Performance for shape identification (from a set of three) was significantly above chance for both active $(84 \%)$ and passive $(56 \%)$ conditions. In the passive condition, the subjects placed their finger on the Optacon and the resulting tactile output from a previous subjects' exploration of the stimuli were played back to them [67].

An early virtual tactile display was the Pantobraille, which combined a planar force feedback device with two standard Braille cells for exploration of a $100 \times 160 \mathrm{~mm}$ workspace [68]. The TVSS developed by the Electronic Vision group in Heidelberg uses a tactile display mounted on a moveable chassis in order to present tactile patterns over a larger surface area of $164 \times 159 \mathrm{~mm}$. The display consists of a pin array constructed from four commercially available Braille cells, mounted together to provide a $6 \times 8$ display, which is a convenient size for three fingers. The tactile display is mounted on a chassis that permits translational movement in the $X$ and $Y$ direction. This permits 2880 pixels in the entire system as the tactile display is swept across the workspace. The device can access visual images across the Internet, stored locally on a computer or through a camera attached to a PC [69].

Gapenne et al. describe a tactile display for the visually impaired that uses a graphics tablet to provide spatial input with the dominant hand, and two commercial Braille cells to provide tactile output to the non-dominant hand [70]. Central to the group's work is the concept of parallelism, which states that the more individual sensor/ stimulator pairs that are simultaneously employed (e.g. individual pins on a tactile display), the more precisely and easily information can be explored and extracted [71]. It is argued that a greater number of sensor/stimulator pairs used in parallel not only enriches the sensations received, but also aids decision making during exploration due to the additional contextual information that is made available [70]. In further experiments, it was demonstrated that supplementing the tactile feedback with audio cues regarding the frame of reference (location of the $x$ and $y$ axes) aided perception of geometric forms, as presenting the axes in a second modality allowed them to be quickly differentiated from the stimuli easily [72].

The first commercially available pin-array augmented mouse was released by Virtouch Ltd (www.virtouch2.com). Intended for tactile presenta-

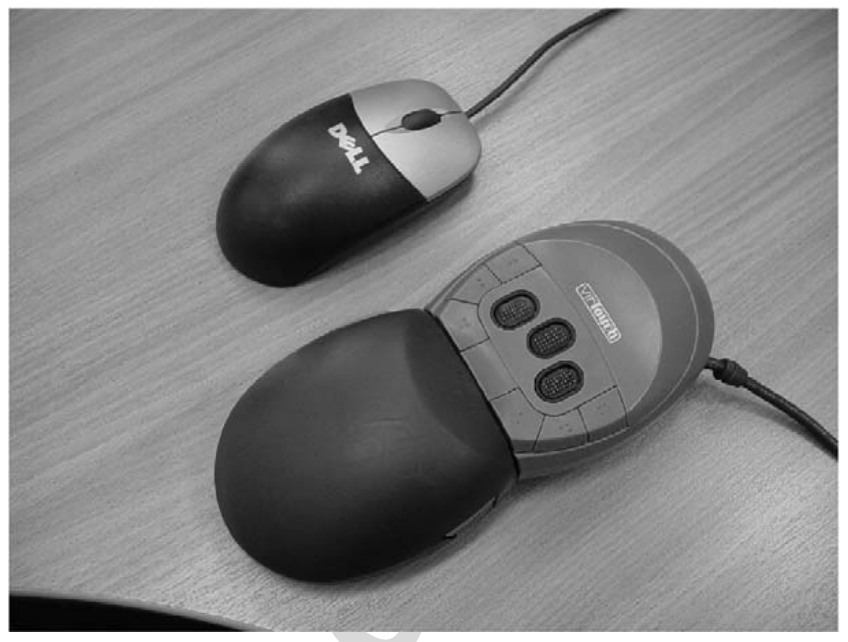

Fig. 5. The VTMouse, an example of a virtual tactile display with three $4 \times 8$ pin arrays, shown with a standard mouse for comparison of size.

tion of graphics to the visually impaired, the first iteration of the device (no longer produced), the VTMouse (Fig. 5), was equipped with three $4 \times 8$ pin arrays on which the user rested his/her fingers during operation (a review of the technical specifications of the device and perceptual experiments can be found in [73]). More recently, this model was superseded by the VTPlayer (Fig. 6), a more compact device with two $4 \times 4$ tactile pin arrays. Jansson and Pedersen describe a study in which blindfolded sighted people and visually impaired users employed the VTPlayer mouse to explore tactile and audio representations of maps of the states of the USA. They compared conditions of audio and multimodal (tactile and audio) feedback. There was no significant difference in performance for multimodal feedback over audio alone, showing that the addition of tactile feedback did not affect the user's performance. However, Jansson and Pederson noted that their participants had little training with the mouse, and problems with the software were noted where there was a lack of auditory feedback in certain situations [74].

\subsection{Tactile-audio sensory substitution}

Work on tactile substitution for the profoundly deaf did not develop much until the late 1970s and early 1980 s, comparatively late compared to tactile vision substitution aids. One of the earliest incarnations of this type of device was the Tacticon, a commercial device that adjusted the perceived intensity of 16 electrodes, each of which corresponded to 


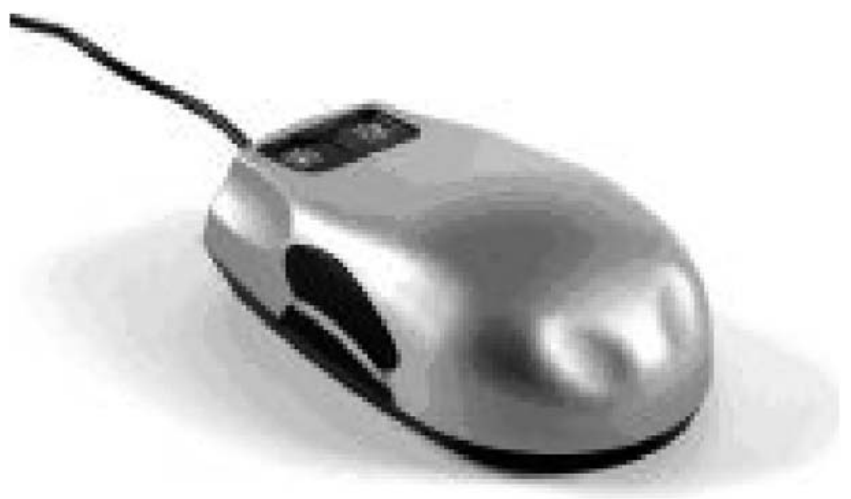

Fig. 6. VTPlayer tactile augmented mouse. A commercially available virtual tactile display with two arrays of $4 \times 4$ pins.

a range of frequencies in the auditory spectrum in order to improve speech comprehension, auditory discrimination, and the clarity of the users speech (results summarised in [9]). Another early device, the Tactile Acoustic Monitor (TAM) was developed in the 1980s at the Biomedical Physics Group of the University of Exeter by Dr Ian Summers, one of the UK's leading exponents of tactile-auditory substitution research. The TAM employed a single vibrotactile stimulator to provide information about the loudness of the user's speech and other sounds in the environment. Sound is picked up by a microphone, which is then compared to a threshold level which can be self-adjusted. If the microphone signal is above the threshold the vibrator is turned on at a constant amplitude and frequency, whereas if the sound level falls below the threshold the vibrator is turned off. A UK Medical Research Council study showed that the TAM was also useful for lip reading applications, prompting a variety of experiments investigating speech perception via a single vibratory transducer (A concise summary exists in [75]). Summers and colleagues have recently developed a pin array tactile display for the fingertip. The device consists of a $10 \times 10$ matrix of pins over an area of $1 \mathrm{~cm}^{2}[6,36]$. At present, the size and weight of the actuators preclude the device from portable applications for investigation of active touch, but the Biomedical Physics Group have produced smaller devices with less stimulators that can be mounted on a mouse to provide a virtual tactile display.

\section{Teleoperation and virtual environments}

Researchers became interested in the idea of computer mediated tactile sensations through the field of teleoperation. Teleoperation is concerned with human control of a remote slave device (typically a robot manipulator arm) using a local master device. The user interacts with the master device, and his/her movements are communicated to the slave, and subsequently replicated. This allows the user access to perform complex manipulation tasks in hazardous environments (underwater maintenance, space exploration, nuclear reactors, bomb disposal) at no personal risk. User control is typically based upon visual feedback cues obtained from a camera at the remote location; however, improved control of the slave manipulator can potentially be achieved by incorporating other feedback modalities, such as force feedback, to provide the operator with an indication of the contact forces encountered by the slave manipulator in the remote environment. Goertz and Thompson developed the very first teleoperation system to include force display at the Argonne National Laboratory. Growth in space exploration led to pivotal work by NASA and the Jet Propulsion Laboratory (JPL) with the introduction of computer-based Cartesian control, which allowed master devices to be smaller and kinematically dissimilar to slave devices (see [10] for an extensive history of force feedback devices). Tactile feedback can be used to relay contact information from the tip of a remote tool or the jaws of a slave manipulator to a human operator. Meaningful tactile feedback may be of great importance for fine control of force and position in grasping and manipulation tasks, and for controlling the level of force exerted at the tip of a tool on a slave device. Motivation for the cross fertilisation of ideas between earlier sensory substitution work and teleoperation arose from the need to provide increased tactile sensitivity to astronauts during extra vehicular activity on space missions. The thick, many-layered protective gloves worn by astronauts preclude tactile information during complex dextrous tasks such as satellite servicing or space vehicle maintenance. Pioneering work in this area was performed by Paul Bach-y-rita and colleagues, who investigated the presentation of sensed forces on the fingers to electrotactile displays on the torso of astronauts [76].

The need to provide tactile cues became more prevalent as more sophisticated master-slave systems were developed for applications such as remote surgery. Shimoga provides a review of tactile feedback technology that could potentially be applied in design of teleoperator systems [77]. The 
review reports the work of Patrick [78] as some of the earliest work applying vibrotactile feedback to an exoskeleton hand master. Patrick's experiments with a vibrating voice coil enhanced hand exoskeleton showed an improvement in mean task error over those of visual feedback alone [78]. Edin et al. [79] showed that a short duration, high-frequency vibration display mounted on a master manipulator can be used to represent slip sensations, and hence trigger a quasi-reflexive grasp force increase by the operator during telemanipulation tasks. In related early work on vibration display, Minsky and colleagues describe the sandpaper system, which presented virtual textures via a two degrees of freedom force feedback joystick [27]. Caldwell and Gosney provide some of the earliest reported work regarding display of remotely sensed texture and slip to a human operator via a data glove enhanced with piezoelectric vibratory displays [35]. Identification of textures from a set of four surfaces with differing amplitude and frequency of texture was excellent (above $90 \%$ recognition rates). Their system was also capable of thermal and pressure sensations.

Motivated by the potential for development of tactile displays for distributed fingertip information in teleoperator and haptic force feedback applications, Lederman and Klatzky performed a series of studies using a rigid fingertip sheath to mask distributed cutaneous information [80]. Two-point discrimination distances were found to be substantially larger (over a 6-fold increase), force detection was significantly impaired, but not as drastically (a 1.73-fold increase in threshold). Vibrotactile detection thresholds were not significantly impaired and subjects could also reliably estimate the roughness magnitudes of tactile raised dot patterns, though discrimination capabilities were significantly diminished compared to without the rigid sheath. Performance in perceptual tasks such as orientation of $2 \mathrm{D}$ bars and detection of a rigid lump via palpation demonstrated significantly less accuracy with the finger sheath. Lederman and Klatzky's results showed that spatial acuity was significantly obviated, but it was still possible to use temporally varying cues in discrimination of vibrotactile thresholds and judgements regarding roughness of surface textures. More recently, Jansson and Monaci demonstrated that providing cutaneous information at the fingertips was of considerably more benefit than increasing the number of limited contact points available with a remote or simulated environment via a haptic interface or teleoperator [81].
Both Cohn et al. [82] and Hasser and Weisenberger [83] performed pioneering work in the area of tactile feedback for teleoperation applications. Hasser and Weisenberger report a prototype $5 \times 6$ element shape memory alloy actuated display. In pilot experiments on pattern identification it was shown that users could reliably identify visual representations of patterns presented tactually with the display. The active elements of the display were driven at frequencies between 5 and $200 \mathrm{~Hz}$, it was observed that subjects were most likely to confuse stimuli based on spatial similarity of patterns, rather than overall pattern density (number of active array elements) [83].

Perhaps the earliest exponents of complete teleoperation systems incorporating both force feedback and pin array displays were Robert Howe and colleagues in the mid-1990s [84]. Kontarinis et al. investigated the effect of tactile sensing and display to a human operator in a telemanipulation task [85]. Output from a tactile sensor located on a remote manipulator was relayed to the operator's fingertips via a tactile pin array of $6 \times 4$ individually controllable elements. Shape memory alloy actuators were used to drive each pin up to a maximum amplitude of $3 \mathrm{~mm}$ (a more extensive description of the device can be found in [42]). A teleoperated hand system for thumb and finger grasp was used for the master and slave manipulators (fully described in [86]). The master and slave device are identical twofingered hands with two degrees of freedom in each finger allowing movement in the vertical plane. Subjects were asked to locate a rigid cylinder inside a block of foam rubber in the absence of visual feedback. With tactile feedback, the subjects were able to locate the cylinder with an error of $3 \mathrm{~mm}$ or less in 57 out of the 60 trials conducted. Conversely, when tactile feedback was not available, the mean absolute error was over $13 \mathrm{~mm}$.

Richard and Cutkosky [87] explored the effects of augmenting force feedback with tactile ("ungrounded") feedback for surface contact. Thus, a single translational degree of freedom haptic interface was augmented with an actuated "force applicator" that provided a contact force at the user's fingertip when they collided with a virtual wall. While there was no quantitative benefit in terms of boundary detection between free-space and haptic constraint, subjects reported enhanced realism of contact sensations.

Building on their earlier expertise with vibrationbased display, Professor Darwin Caldwell and 
colleagues at Salford University developed a more advanced tactile display based on pneumatically controllable pins for display of local shape and texture to an operator's fingertip in a telemanipulation task [38]. A series of informal evaluations were undertaken with the display attached to a data glove. Users reported a good ability to detect and track edges using the shape display and were able to discriminate textures based on their frequency and amplitude characteristics. The authors report further in depth tests as on going in this area.

\section{Tactile display for multimodal human-computer interaction}

The mid-1990s also saw tactile displays used within desktop interaction for the first time. Little of this work focussed on tactile pin arrays; rather, single pin or vibrotactile output was more common. The aim of this work generally has been to supplement the graphical feedback from current computer systems with another form of output. This is often done because the visual sense is overloaded and users can miss information if presented graphically; the tactile sense is underutilised and so is available for information presentation. It is also the case that tactile feedback might be more appropriate than graphics for communicating certain types of information.

Much of the focus of work in this area to date has been on device and hardware development; until recently there were few tactile transducers routinely available and they were often designed specifically for use in sensory substitution or teleoperation, making them impractical as everyday devices for desktop interaction. Now many mobile telephones and hand held computers have vibrotactile actuators included for alerting. Lee et al. [88] have also recently developed a tactile stylus to use on touch screens and hand held computers. Poupyrev et al. and Fukumoto et al. [89-91] have designed sophisticated tactile displays for hand held computers. These have enabled research to begin into how best to use touch for interaction.

Significant early work in tactile human-computer interaction was done by Akamatsu and colleagues who investigated the addition of an electromagnetically controlled pin to a standard mouse. This allowed users to feel vibrations through a fingertip. Akamatsu, MacKenzie and Hasbrouc [92] investigated the impact of their tactile feedback on target acquisition in a Fitt's law study [93]. They examined whether targeting was aided when sound, tactile and colour feedback were used to indicate target acquisition in a desktop-type pointing interaction. They compared five conditions: 'normal', the three feedback modes in isolation, and a 'combined' condition in which all feedback modes were used simultaneously. Although they found no significant difference in overall selection time, their results showed that target highlight time was reduced when feedback was present. Tactile feedback appeared to have a greater effect in reducing highlight time than either sound or colour.

In a second study, Akamatsu and MacKenzie [94] examined the contribution of tactile and forcefeedback in targeting. They found a significant difference between normal, tactile, force, and force + tactile feedback conditions. Tactile and force + tactile feedback reduced targeting times by $5.6 \%$ and $7.6 \%$, respectively. Force-feedback alone resulted in slightly higher targeting times. The authors did not consider multiple targets and the distractions that tactile feedback might cause in more complex, real-world displays with many targets on a display presenting un-needed feedback as the user moves over them.

There has been less research into the use of tactile feedback in more complex and realistic, multiple target displays. Vitense et al. [95] investigated how all combinations of visual, audio and tactile feedback influenced user performance in drag-and-droptype tasks. The feedback modes were activated when the dragged object was over the target. Tactile feedback was provided by vibrating a Logitech Wingman force feedback mouse. Dependent measures included the total trial time, the highlight time (between entering the target and dropping the object), and subjective responses. There were several interesting results, including the fact that tactile feedback increased the total trial time but reduced the target highlight time. Overall, their results showed that some combinations of feedback were very effective, but other combinations were not and actually reduced performance. This study was based on a more realistic task than some of the others done previously, but there were still few distracter targets for users to cross on the way to the item of interest.

Cockburn and Brewster [96] looked at combinations of different feedback modalities, including vibration feedback from a Logitech iFeel mouse (www.logitech.com), for selecting small targets on a computer desktop. They found that, in simple Fitt's 
law-type tasks (where discrete targets are used, so there are no distracters), tactile and audio feedback both reduced targeting time (confirming Akamatsu's results above), but the combination of audio plus tactile was not as good as when each was used alone. However, in a more realistic task (choosing items from drop down menus) the tactile feedback caused problems and actually increased targeting time over a standard graphical display. The reason for this was the close proximity of many tactile targets (each menu item gave tactile feedback) causing feedback 'overload'. As the user moved over a menu to get to a specific item, all the items traversed gave tactile feedback which distracted the user. Cockburn and Brewster suggest that " ... care should be taken to make sure that feedback suits the particular task. It may be that feedback good in one situation is poor in another due to excessive distraction". They give some suggestions as to how some of the problems of distracters can be avoided so that the advantages of tactile target acquisition can be retained in complex interactions.

Many of the studies described above used very simple tactile cues, for example, vibrating a mouse to indicate that a target has been reached. Brewster and Brown have been investigating more complex cues which encode more information. They have proposed Tactons, or tactile icons. These are structured, abstract messages that can be used to communicate tactually $[22,23]$. Information is encoded into Tactons using the basic parameters of cutaneous perception, such as waveform, rhythmic patterns and spatial location on the body. Results have shown that information can be encoded effectively in this way and that users can easily extract the information.

Brewster and King [97] looked at using simple Tactons to present progress information tactually. Progress bars are common in graphical user interfaces of all types, from desktop systems to mobile phones. They compared a standard visual progress bar to a tactile one. The tactile progress bar indicated progress information by the time between two tactile pulses; as the download progressed, the pulses got closer together, eventually overlapping when the download completed. They showed that, if users were engaged in a visually demanding task like typing, the tactile display could indicate progress significantly more effectively than the standard visual one, as it did not compete for visual attention with the typing task. This again shows that tactile displays have much untapped potential for improving human-computer interactions.

\section{Experiment}

Tactile displays clearly offer great potential for enabling visually impaired people access to digitally stored and manipulated data. The most commonly employed solutions for access at present include screen readers, screen magnifiers, Braille displays, and producing tactile diagrams with heat-raised paper. There are several drawbacks with these methods in that they are either unable to respond quickly to dynamic changes in data (hard copies need to be produced of heat-raised diagrams), they are inherently serial in nature and therefore highly memory intensive (e.g. a screen reader or Braille display representing numerical data from a large table), use an abridged form of data (pre-recorded descriptions of graphs or Braille versions of tables), or are simply inaccessible to potential users (only $26 \%$ of visually impaired university students read Braille, and screen magnifiers are useless to those with no residual vision).

A virtual tactile display (see Section 4.2) could potentially make graphical representations of large data sets more accessible to visually impaired people using tactile representations of common visualisations such as line graphs, bar charts and pie charts. The purpose of this study was to investigate the effectiveness of a commercially available, mousebased tactile display for the purposes of representing data in the form of a line graph. The study focussed on one of the most fundamental components of comprehending data presented as a line graph: perception of the gradient of lines.

\subsection{Equipment}

The device used in the study was the VTPlayer tactile mouse (Fig. 6) which is similar to a standard computer mouse, except that the user rests their index and middle fingers on the two tactile displays located on top of the chassis. The displays both consist of a $4 \times 4$ matrix of pins that are each individually controllable through software. Pins can either be raised or lowered; under standard operation, the states of the pins are related to the colour of the pixels surrounding the mouse pointer - a dark pixel corresponding to a raised pin and a light pixel to a lowered pin (exact thresholds are unknown). Thus, a user can actively explore a 
desktop, menu, or other on-screen environment and receive tactile information regarding what is under the mouse pointer.

Exploring a complex environment, such as a desktop or other visually oriented graphical user interface, is extremely difficult from tactile information alone. Thus, for the purposes of the study, some simple line stimuli were created, consisting of black lines on a white background. When exploring the lines with the VTPlayer, the lines were represented by raised pins on a background of lowered pins. The experiment utilised two data sets, each using lines of different thickness. From informal observations, it seemed that using thicker lines may make them easier to locate, but may also obscure gradient information due to the limited resolution of the VTPlayer's tactile displays.

\subsection{Previous research}

Previously at Glasgow University, researchers have studied the perception of line gradient using both traditional heat-raised paper diagrams used by visually impaired people, and also with an inexpensive force feedback mouse (Logitech's Wingman) [98]. Performance, in terms of identifying positive and negative gradients relative to a horizontal line, was very accurate, and comparable, for both media. Performance was at ceiling level within roughly 6 degrees of the horizontal. This illustrated that people could perform the task in the absence of tactile cues, using kinaesthetic information from the force feedback mouse. Studies in the psychological literature have focussed on particular phenomena, such as the "haptic oblique effect", which shows reduced accuracy in oblique line orientations compared to vertical or horizontal $[99,100]$.

\subsection{Experimental procedure}

Twelve participants were recruited for the experiment from the student population at the University of Glasgow. All participants were sighted and righthanded. The stimuli consisted of a set of 19 images of lines which the participants were to explore using the VTPlayer. The range of angles used in the data set was obtained using informal pilot studies with members of the authors' research group. The stimuli consisted of lines between $-24^{\circ}$ and $+24^{\circ}$ (relative to the horizontal) at stepwise angular increases of $3^{\circ}$. There was also a line at $-30^{\circ}$ and $+30^{\circ}$ relative to the horizontal. Thus, the full set of lines were oriented at the following angles: $-30^{\circ},-24^{\circ},-21^{\circ}$, $-18^{\circ},-15^{\circ},-12^{\circ},-9^{\circ},-6^{\circ},-3^{\circ}$ (all sloping down), $0^{\circ}$ (horizontal), $3^{\circ}, 6^{\circ}, 9^{\circ}, 12^{\circ}, 15^{\circ}, 18^{\circ}, 21^{\circ}, 24^{\circ}, 30^{\circ}$ (all sloping upwards). The full data set for the experiment consisted of 4 repetitions of each of these lines, giving a set of 76 lines. The stimuli were generated using Microsoft Excel, and copied in to bitmap files of size $370 \times 345$ pixels (approx $11 \mathrm{~cm}$ by $10.2 \mathrm{~cm}$ ). Two sets of lines were generated, using different line thickness, to test for the effect of this on perception of gradient. The "thick" lines were 3 pixels in thickness. The "thin" lines were 2 pixels in thickness. All the lines were approximately $5 \mathrm{~cm}$ in length and ran through the centre of the bitmap image. During the experiment, the user's mouse movements were constrained by the VTPlayer software to an area around the centre of the bitmap of $290 \times 265$ pixels, as the edge of the bitmap contained no information and made it harder for participants to locate the stimuli.

There were two conditions in the experiment, "thick lines" and "thin lines". The order in which the participants attempted these conditions was counterbalanced between them in order to counter for ordering effects, thus, half the participants attempted the "thick lines" condition first, and the other half attempted the "thin lines" condition first. Participants explored the lines using the VTPlayer with their dominant hand (in this case, the right hand, for all participants). The cursor speed of the VTPlayer was scaled to one-tenth that of normal operation for the purposes of the experiment. It was found during piloting the experiment that novice VTPlayer users tended to move the mouse very quickly, which resulted in the line stimulus being moved over in between updates of the tactile display, thus it was very easy for participants to miss the stimulus and become confused. The participants' task was to indicate whether the line sloped upwards or downwards from left-to-right. There was no time limit imposed for this task. Participants logged their answer by pressing a key on the keyboard using their non-dominant hand. The participants were instructed to guess at the answer if unsure, as some of the stimuli may be close to the limits of their perception. Participants were not blindfolded, as they were required to follow onscreen instructions and use the keyboard to record their answers, however, their dominant hand and the mouse were hidden from view behind a screen (see Fig. 7). 


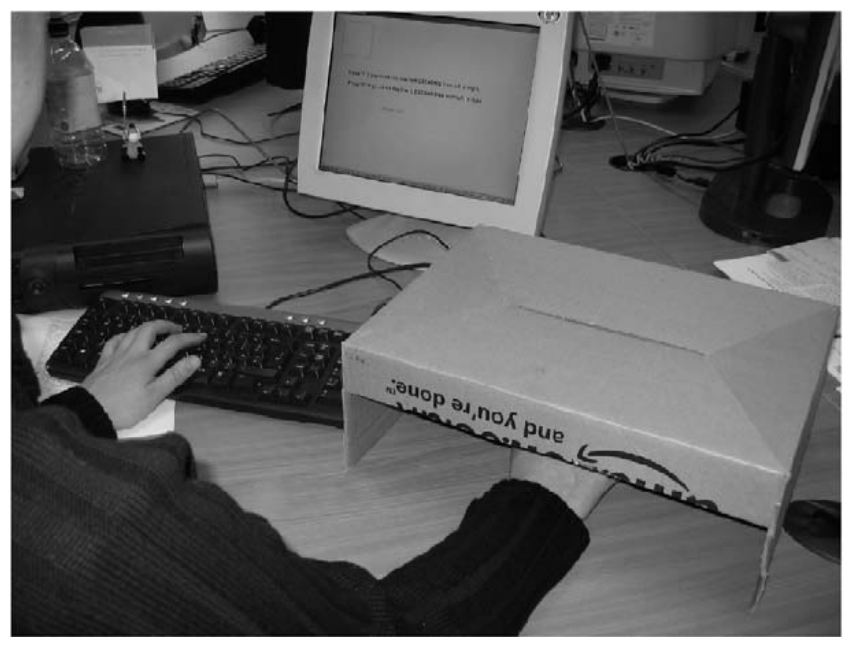

Fig. 7. Experimental set-up for line discrimination study. The participant operates the VTPlayer with their dominant hand behind the screen, and logs responses on the keyboard with the non-dominant hand.

During each step of the experiment the participant was presented with one of the stimuli to explore using the VTPlayer. The position of the mouse was reset to the centre of the bitmap containing the stimuli before each trial, using the VTPlayer software, to make the line stimuli easy to locate for the participant. During exploration of the stimuli, the participant received no on-screen visual feedback from a mouse pointer, so that their judgements were based purely on kinaesthetic information from mouse movements, and cutaneous stimulation from the VTPlayer's tactile displays. Upon making a decision, a visual on-screen warning instructed the participant to hit the return key when ready to receive the next stimuli. While this dialog box was on-screen they were free to take a break if needed, and the mouse input and output was disabled. The order of presentation of stimuli was randomised for each participant. Participants were given 19 practice stimuli (one of each unique line) before participating in the experiment. During this practice session, the mouse cursor was visible to the participants (they were informed prior to the practice session that they would receive no visible feedback during the controlled experimental conditions); this was so the participants could learn the relationships between their hand movements and the movement of the mouse pointer, and for them to learn strategies of hand movements to locate and explore the line stimuli.

\subsection{Results}

For the purposes of analysis, the proportion of times that participants responded that a line was increasing from left-to-right was recorded. Representing data using this method (as opposed to, say, proportion of correct responses) allowed us to apply standard psychophysical methods in order to calculate the difference threshold for correct identification of gradient. Initially, statistical analysis was performed on both the "thick lines" and "thin lines" data set using an ANOVA. Statistical analysis was performed using the Minitab software (www.minitab.com). The dependant variable was the proportion of "increasing" responses averaged over all subjects. The independent variables were the stimulus angle and the thickness of the lines. It was found that the angle of the line had a highly significant effect on the participants' responses $(\mathrm{F}(18,18)=271.3, P<0.001)$, and that the thickness of the line also had a significant effect $(\mathrm{F}(1,18)=6.04, \quad P<0.05)$. Fig. 8 illustrates the relationship between the angle of the stimulus, the thickness of the lines and the proportion of times the line was identified as "increasing" by the participants. The graph indicates a difference in performance for negative gradients, with thin lines being identified as "increasing" more regularly, thus performance was less accurate for the thin lines. However, for positive gradients the data for both

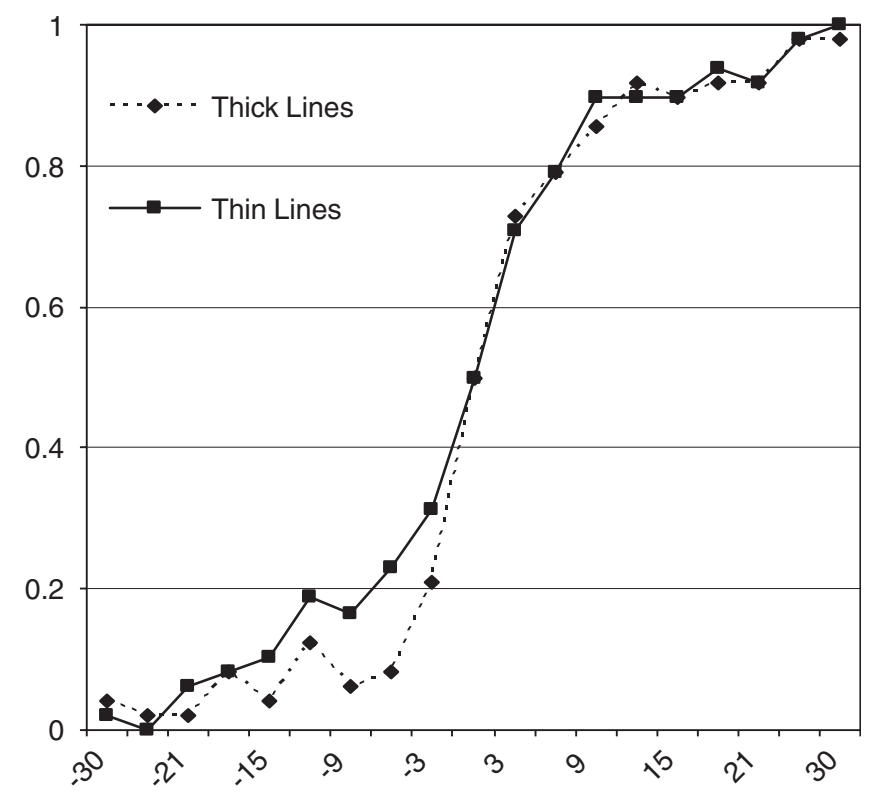

Fig. 8. Proportion of times "increasing" answer was given in this experiment, as a function of stimulus angle, for "thick lines" and "thin lines". 
thicknesses of lines appear to be similar. This was investigated by performing a two-tailed $T$-test for negative and positive angles separately. In both cases the dependant variable was line thickness and the independent variable was proportion of times "increasing" was given as the answer. The $T$-test for negative gradients verified that there was a significant difference between the two line thicknesses ( $T=2.31, P=0.027)$, confirming that performance was significantly less accurate for thin lines. The $T$-test for positive gradient data showed that in this case there was no significant difference between the two different line thicknesses $(T=2.31, P=0.51)$. As the thickness of the lines only had a significant effect for negative gradients, the two data sets were collapsed in to a single data set for purposes of further analysis. Fig. 9 shows the proportion of positive responses for each angle averaged over both thickness conditions and all subjects.

The data are approximate to a cumulative normal curve (an ogive), the typical psychometric function for a difference threshold experiment. The data were converted to $z$-scores from the normal distribution. When the proportion of responses is expressed as $z$ scores, the cumulative normal distribution becomes a linear function. Hence, by fitting a straight line to the data when plotted against $z$-scores, the best-fit cumulative normal model can be found for the data (see solid line in Fig. 9). The model was fit using data only in between $\pm 9^{\circ}$, as this was the most salient for calculating difference thresholds. The model was initially fit to the entire data set, but the ceiling effects observed above this limit skewed the model, providing a poor estimate of data around the threshold. The upper difference threshold (DL) corresponds to $75 \%$ response rate, hence the upper DL predicted by the model is $4.7^{\circ}$. The lower DL corresponds to $25 \%$, which corresponds to $-4.39^{\circ}$ as predicted by the model.

The data from Riedel and Burton's study [98] which employed the same methodology with raised paper stimulus and a force feedback mouse was obtained and re-analysed using the above method. Cumulative normal curves were fit to the data, as shown in Fig. 9. The data for both force feedback and raised paper showed a significant constant error (CE) between the point of subjective equality (PSE) and the horizontal $\left(0^{\circ}\right)$, when analysed using the method described above. Thus the PSE was fixed at $0^{0}$ when calculating the models for the data. This allowed for a better comparison with the data obtained from the VTPlayer in terms of the slope of the psychometric functions, and the upper and lower DLs. The DL for raised paper was calculated as $\pm 2.42^{\circ}$. The model predicted that the DL with the force feedback mouse was $\pm 3.25^{\circ}$.

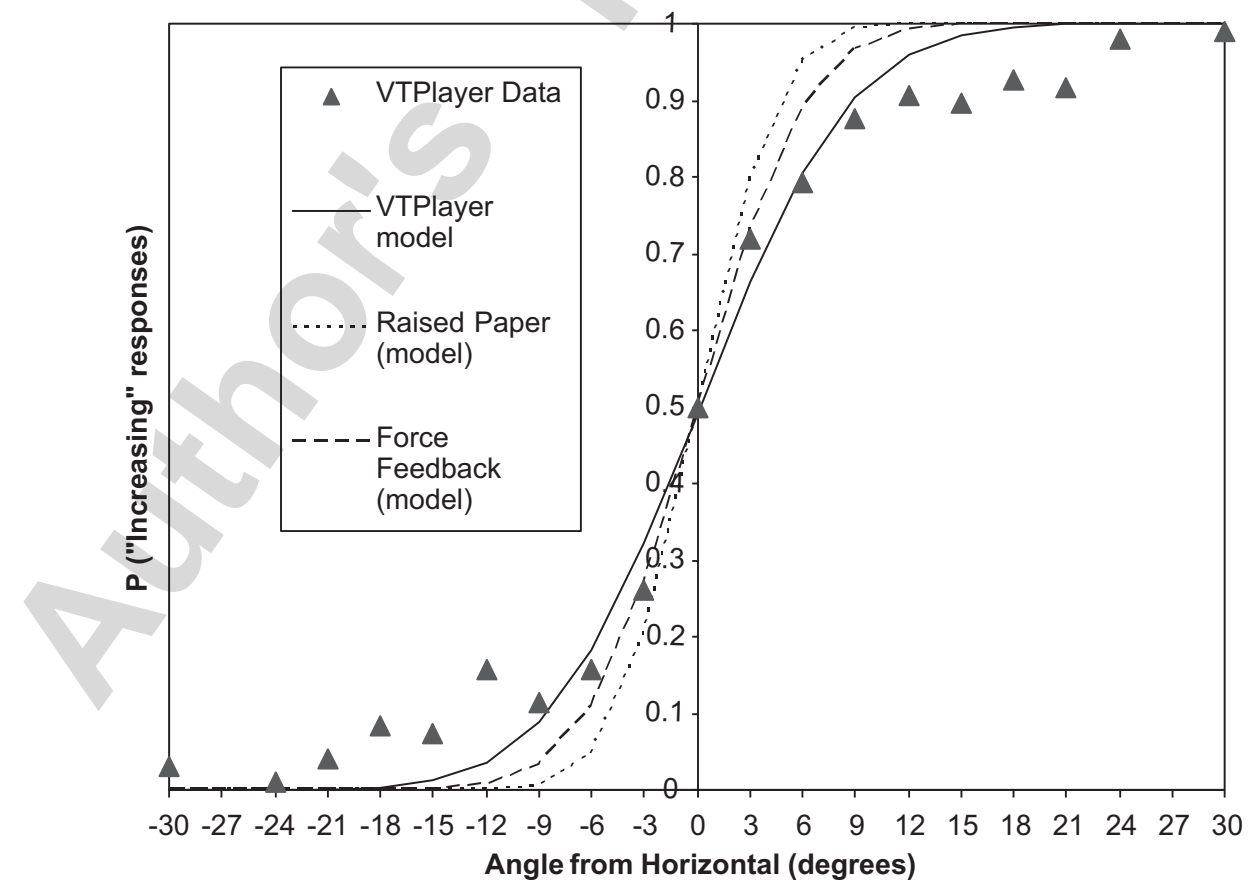

Fig. 9. Proportion of times "increasing" answer was given, as a function of stimulus angle and method of representation. Comparison between VTplayer tactile mouse, raised paper diagram and force feedback mouse results. 


\subsection{Discussion}

Comparing the results for the tactile mouse alongside the results for the force feedback mouse will allow conclusions to be drawn regarding the relative contribution of tactile and kinaesthetic cues in the line gradient discrimination task. Using the raised paper diagrams currently provides the richest combination of tactile and kinaesthetic cues, while allowing the user to employ both hands and several fingers for exploration, and is thus the benchmark for discrimination tasks. As can be seen from Fig. 9, the psychometric curve for raised paper is the steepest; therefore the observer's differential sensitivity is finest in this condition. The models also suggest that users of the force feedback mouse were also more able to perform the gradient discrimination task than those using the tactile mouse. In the real-world task using the raised paper graphs, a large component of the task of identifying the gradient is detection of edges and contours of the raised lines. Studies have shown that performance in edge detection is degraded when the explorer is deprived of distributed tactile cues on the fingertip by interposing a rigid sheath between the finger and the stimulus [80]. Thus, it could be concluded that tactile stimulation of the skin is important to perform this task well. However, the tactile mouse users performed worse at the task than the force feedback users. Previous studies have shown that force feedback is good for constraining and guiding a user along a particular path, which may account for the good performance with the force feedback mouse [16]. Several factors differ between the stimulation obtained from touching a surface directly, and that arising from the matrix of stimulators on the tactile mouse. Firstly, the resolution of the mouse is quite poor compared to the resolution of the mechanoreceptors in the human fingertip. Secondly, only a limited population of the receptors will be stimulated by the action of the mouse. In particular, the SAII afferents are less sensitive to skin indentation, but more sensitive to lateral skin stretch [43] and would therefore not be stimulated by the motion of the tactile displays. Finally, as discussed in Section 4, the update rate of the mouse display is often not fast enough to allow exploration of the stimuli if quick movements are made. From the methodology used in this study it is impossible to isolate these effects, but it is likely that they all contributed in some way to the degradation in performance with the tactile mouse when compared to direct finger contact with raised paper stimuli.

\subsection{Conclusion}

Users of the tactile mouse could successfully discriminate positive and negative gradients within roughly $\pm 5^{\circ}$ of a horizontal line. This performance was worse than difference thresholds predicted by models derived from results for a similar experiment using raised paper lines, and a force feedback mouse. It appears that the kinaesthetic cues provided by force feedback are a more salient cue than the distributed tactile stimulation provided by the VTPlayer tactile mouse's displays. The displays are, however, of a limited resolution and bandwidth, which will have contributed in some way to the result.

\section{Future work and research challenges}

\subsection{Technology}

Future developments in tactile display technology will likely lead to devices of reduced size and cost and increased resolution of pins (smaller spacing between adjacent elements). In particular, for virtual environment and teleoperation applications seeking to create realistic, simulated textures, displays of a resolution approaching that of the human sensory system will be necessary to create realistic feeling textures. Reducing the size and cost of devices will allow them to be more easily and cheaply incorporated in to mechanisms with force feedback. One of the most important perceptual cues in tactile embossed representations is the use of height for demarcation of differentially salient areas on a diagram, as noted by Challis and Edwards [20]. Therefore, an important technological output of future research will be a tactile array capable of sufficient displacement and control of pins to allow features of different amplitudes to be rendered. This could be used, for example, to tactually distinguish the content of a graph from its axes.

\subsection{Human factors}

Much of the perceptual and psychophysical information available in the literature has been conducted with vibrating pin arrays, such as the Optacon, as opposed to displays for skin indentation. Therefore, along with the development of 
devices, work is also needed on the perceptual issues of static displays. Guidelines need to be developed from psychophysical results for device parameters such as display size, pin resolution, actuator bandwidth and force output. More understanding of perceptual phenomena such as spatial and temporal masking of cues presented by pin arrays is also needed to help designers develop applications and tactile representations.

The commercial availability of virtual tactile displays, such as the VTPlayer, that allow active exploration of a larger image provide an ideal tool to evaluate the relative contribution of kinaesthetic and cutaneous information when interpreting tactile diagrams. By adopting an experimental paradigm based on modes of active and passive touch it is possible to isolate the contribution of both channels. These results will be important for developers, as, if tactile information can be communicated by passively received cutaneous information, this will free the need for devices to provide kinaesthetic cues, allowing them to be made smaller and more portable.

\subsection{Applications}

Perhaps the highest motivating factor for research into tactile displays is now the simulation of realistic, haptic virtual environments (VEs) that combine force feedback with tactile displays. However, force feedback interfaces need to be improved to match the movement range, force capabilities and resolution of human movement and sensing. Srinivasan and Basdogan [2] suggest that once tactile displays are developed and integrated with sophisticated force feedback mechanisms "we can expect an explosion in the applications of haptics in VEs". A deeper understanding is required of how users perceive and interpret cues from a tactile display in conjunction with force feedback. Rendering algorithms also need to be developed in order to ensure consistency of representation on both devices. For display of remotely sensed environments for teleoperators there is also the challenge of developing sensors of sufficient resolution capable of detecting pressure, slip and vibration to relay to the operator.

Tactile access to graphically stored data for the visually impaired still needs to be made affordable, discrete and portable, as well as accessible. Virtual tactile displays could potentially provide dynamic access to digitally stored information such as graphs, maps and charts that are currently only available in an abridged or static format. Guidelines and standards for designers for the presentation of tactile cues need to be established, along with how best to combine tactile display with other modalities such as auditory display. As Jansson notes, mousebased devices such as the VTPlayer may not be the most appropriate for the visually impaired, as they rely on spatially distributed cues to guide exploration [74], therefore other input devices will need to be investigated. Haptic force feedback displays [16] provide a spatially grounded frame of reference for exploration and force cues for guidance, but they are often bulky and expensive. Graphics tablets $[59,71]$ provide the benefit of a spatial frame of reference and are relatively inexpensive for the individual, but more research is required to validate their use with tactile output. Bimodal combinations of tactile and auditory information [14,20,21,72] have been demonstrated to offer numerous benefits to visually impaired users. Establishing guidelines for assigning cues to modalities would help designers develop applications.

\section{References}

[1] T. Massie, K. Salisbury, The phantom haptic interface: a device for probing virtual objects, ASME Annual Winter Meeting, New York, 1994, pp. 295-300.

[2] M.A. Srinivasan, C. Basdogan, Haptics in virtual environments: taxonomy, research status, and challenges, Comput. Graph. 21 (4) (1997) 393-404.

[3] M. Govindaraj, A. Garg, A. Raheja, G. Huang, D. Metaxas, Haptic simulation of fabric hand, Eurohaptics, Dublin, Ireland, 6-9 July 2003, pp. 253-260.

[4] P. Dillon, W. Moody, R. Bartlett, P. Scully, R. Morgan, C. James, Sensing the fabric: to simulate sensation through sensory evaluation and in response to standard acceptable properties of specific materials when viewed as a digital image, in: S. Brewster, R. Murray-Smith (Eds.), Haptic Human-Computer Interaction, Springer, Heidelberg, 2001, pp. 205-217.

[5] S. Baillie, A. Crossan, S. Reid, S. Brewster, Preliminary development and evaluation of a bovine rectal palpation stimulator for training veterinary students. Cattle Practice, J. Br. Cattle Vet. Assoc. 11 (2) (2003) 101-106.

[6] I.R. Summers, C.M. Chanter, A.L. Southall, A.C. Brady, Results from a tactile array on the fingertip, Eurohaptics, Birmingham, UK, 1-4 July 2001, pp. 26-28.

[7] J.C. Craig, Tactile pattern perception and its perturbations, J. Acoust. Soc. Am. 77 (1) (1985) 238-246.

[8] J.C. Craig, C.E. Sherrick, Dynamic tactile displays, in: W. Schiff, E. Foulke (Eds.), Tactual Perception: A Sourcebook, Cambridge University Press, Cambridge, 1982, pp. 209-233.

[9] K.A. Kaczmarek, P. Bach-y-rita, Tactile displays, in: W. Barfield, T.A. Furness, (Eds.), Virtual environments and 
advanced interface design, Oxford University Press, Inc., New York, 1995.

[10] G.C. Burdea, Force and Touch Feedback for Virtual Reality, Wiley, New York, NY, 1996.

[11] C. Sjostrom, Using haptics in computer interfaces for blind people, CHI 2001, Seattle, WA, 31 March-5 April 2001, pp. 245-246.

[12] J.P. Fritz, K. Barner, Design of a haptic graphing system, 19th RESNA Conference, Salt Lake City, UT, 1996.

[13] E. Wies, J. Gardner, S. O'Modhrain, V. Bulatov, Webbased touch display for accessible science education, in: S. Brewster, R. Murray-Smith (Eds.), Haptic hci, Springer, Lecture Notes in Computer Science, Berlin, 2001, pp. 52-60.

[14] W. Yu, S. Brewster, Comparing two haptic interfaces for multimodal graph rendering, IEEE VR2002, 10th Symposium on Haptic Interfaces for Virtual Environment and Teleoperator Systems, Orlando, FL, 2002.

[15] W. Yu, D. Reid, S.A. Brewster, Multimodal virtual reality versus printed medium in visualization for blind people, Proceedings of ACM ASSETS, Edinburgh, Scotland, 2002, pp. 57-64.

[16] W. Yu, S.A. Brewster, Evaluation of multimodal graphs for blind people, J. Universal Access Inform. Soc. 2 (2) (2003) 105-124.

[17] E. Foulke, Reading Braille, in: W. Schiff, E. Foulke (Eds.), Tactual Perception: A Sourcebook, Cambridge University Press, Cambridge, 1982, pp. 168-208.

[18] W. Gaver, The sonicfinder: an interface that uses auditory icons, Hum-Comput. Interact. 4 (1989) 67-94.

[19] H. Petrie, V. Johnson, P. McNally, S. Morley, A.M. O'Neill, D. Majoe, Inexpensive tactile interaction for blind computer users: two application domains, IEE Colloquium on Developments in Tactile Displays, London, UK, 21 January 1997, pp. 2/1-2/3.

[20] B.P. Challis, A.D.N. Edwards, Design principles for tactile interaction, in: S. Brewster, R. Murray-Smith (Eds.), Haptic hci 200, Springer, Berlin, Heidelberg, 2001, pp. 17-24.

[21] L.R. Wells, S. Landau, Merging of tactile sensory input and audio data by means of the talking tactile tablet, Eurohaptics 2003, Dublin, Ireland, 2003, pp. 414-418.

[22] S. Brewster, L.M. Brown, Tactons: structured tactile messages for non-visual information display, 5th Australian User Interface Conference (AUIC2004), Dunedin, New Zealand, 2004.

[23] L.M. Brown, S. Brewster, H. Purchase, A first investigation into the effectiveness of tactons, World Haptics 2005, Pisa, Italy, 2005.

[24] J. Linjama, T. Kaaresoja, Novel, minimalist haptic gesture interaction for mobile devices, third Nordic Conference on Human-computer Interaction, Tampere, Finland, 2004, pp. $457-458$.

[25] R.L. Klatzky, S.J. Lederman, Tactile roughness perception with a rigid link interposed between skin and surface, Percept. Psychophys. 61 (4) (1999) 591-607.

[26] J.M. Loomis, Distal attribution and presence, Presence: Teleoperator. Virtual Environ. 1 (1) (1992) 113-119.

[27] M. Minsky, M. Ouh-Young, O. Steele, F. Brooks Jr., M. Behensky, Feeling and seeing: issues in force display, Comput. Graph. 24 (2) (1990) 235-243.

[28] T.H. Massie, Initial Haptic Exploration With the Phantom: Virtual Touch Through Point Interaction, Massachussets Institute of Technology, Cambridge, MA, 1996.
[29] J.P. Fritz, K.E. Barner, Stochastic models for haptic texture, SPIE International Symposium on Intelligent Systems and Advanced Manufacturing, 1996, pp. 34-44.

[30] C. Basdogan, C. Ho, M.A. Srinivasan, A ray-based haptic rendering technique for displaying shape and texture of 3D objects in virtual environments, Proceedings of the ASME Dynamic Systems and Control Division, 1997, pp. 77-84.

[31] S.A. Wall, W.S. Harwin, Modelling of surface identifying characteristics using Fourier series, Proceedings of ASME International Mechanical Engineering Congress: Dynamic Systems and Control Division (Haptic Interfaces for Virtual Environments and Teleoperator Systems), Nashville, TN, 1999, pp. 65-71.

[32] S. Choi, H.Z. Tan, An analysis of perceptual instability during haptic texture rendering, 10th International Symposium on Haptic Interfaces for Virtual Environment and Teleoperator Systems (IEEE VR 2002), 2002, pp. 129-136.

[33] K.A. Kaczmarek, J.G. Webster, P. Bach-y-rita, W.J. Tompkins, Electrotactile and vibrotactile displays for sensory substitution systems, IEEE Trans. Biomed. Eng. 38 (1991) 1-15.

[34] L.A. Jones, M. Berris, Material discrimination and thermal perception, 11th Symposium on Haptic Interfaces for Virtual Environment and Teleoperator Systems, Los Angeles, CA, 22-23 March 2003, pp. 171-178.

[35] D.G. Caldwell, C. Gosney, Enhanced tactile feedback (teletaction) using a multi-functional sensory system, IEEE International Conference on Robotics and Automation, 2-6 May 1993, pp. 955-960.

[36] I.R. Summers, C.M. Chanter, A broadband tactile array on the fingertip, J. Acoust. Soc. Am. 112 (5) (2002) 2118-2126.

[37] J. Pasquero, V. Hayward, Stress: A practical tactile display with one millimeter spatial resolution and $700 \mathrm{~Hz}$ refresh rate, Eurohaptics, Dublin, Ireland, 6-9 July 2003, pp. 94-110.

[38] D.G. Caldwell, N. Tsagarakis, C. Giesler, An integrated tactile/shear feedback array for stimulation of finger mechanoreceptor, IEEE International Conference on Robotics and Automation, Detroit, MI, May 1999, pp. 287-292.

[39] J.M. Loomis, S.J. Lederman, Tactual perception, in: K. Boff, L. Kaufman, J. Thomas (Eds.), Handbook of Perception and Human Performance, Wiley, New York, 1986, pp. 31-41.

[40] K.O. Johnson, S.S. Hsiao, Neural mechanisms of tactual form and texture perception, Annu. Rev. Neurosci. 15 (1992) 227-250.

[41] R.S. Johansson, U. Landstrom, R. Lundstrom, Responses of mechanoreceptive afferent units in the glabrous skin of the human hand to sinusoidal skin displacements, Brain Res. 244 (1982) 17-25.

[42] D.A. Kontarinis, R.D. Howe, Display of High Frequency Tactile Information to Teleoperators, 1993, pp. 40-50.

[43] K.O. Johnson, Neural basis of haptic perception, in: H. Pashler, S. Yantis (Eds.), Stevens Handbook of Experimental Psychology, Wiley, New York, 2002, pp. $537-583$.

[44] R.T. Verillo, Effect of contactor area on vibrotactile threshold, J. Acoust. Soc. Am. 35 (1963) 1962-1966.

[45] C.H. Rogers, Choice of stimulator frequency for tactile arrays, IEEE Trans. Man Machine Systems MMS- 11 (1970) 5-11. 
[46] S.J. Bolanowski, G.A. Gescheider, R.T. Verillo, C.M. Checkosky, Four channels mediate the mechanical aspects of touch, J. Acoust. Soc. Am. 84 (5) (1988) 1680-1694.

[47] C.E. Sherrick, R.W. Cholewiak, A.A. Collins, The localization of low- and high-frequency vibrotactile stimuli, J. Acoust. Soc. Am. 88 (1) (1990) 169-179.

[48] J.R. Phillips, K.O. Johnson, Neural mechanisms of scanned and stationary touch, J. Acoust. Soc. Am. 77 (1) (1985) 220-224.

[49] R.S. Johansson, A.B. Vallbo, Detection of tactile stimuli. thresholds of afferent units related to psychophysical thresholds in the human hand, J. Physiol. 297 (1979) 405-422.

[50] J. Lofvenberg, R.S. Johansson, Regional differences in sensitivity to vibration in the glabrous skin of the human hand. Brain Res. 301 (1984) 65-72.

[51] F.A. Geldard, The Human Senses, Wiley, NY, 1972.

[52] G. Werner, V.B. Mountcastle, Quantitative relations between mechanical stimuli to the skin and neural responses evoked by them, in: D. Kenshalo (Ed.), The Skin Senses, Charles C. Thomas, Springfield, IL, 1968, pp. $112-138$.

[53] H.Z. Tan, Information transmission with a multi-finger tactual display, Electrical Engineering and Computing science, Massachussetts, Institute of Technology: Cambridge, MA, 1996.

[54] G.D. Goff, Differential discrimination of frequency of cutaneous mechanical vibration, J. Exp. Psychol. 74 (1967) 294-299.

[55] F.A. Geldard, Adventures in tactile literacy, Am. Psychol. 12 (1957) 115-124.

[56] P.B.L. Meijer, An experimental system for auditory image representations, IEEE Trans. Biomed. Eng. 39 (2) (1992) $112-121$

[57] J.W. Hill, Limited field of view in reading letter shapes with the fingers, in: F.A. Geldard (Ed.), Cutaneous Communication Systems and Devices, Psychonomic Society, Austin, TX, 1974, pp. 95-105.

[58] J.C. Bliss, M.H. Katcher, C.H. Rogers, R.P. Shephard, Optical-to-tactile image conversion for the blind, IEEE Trans. Man Machine Systems MMS- 11 (1970) 58-64.

[59] J.M. Loomis, R.L. Klatzky, S.J. Lederman, Similarity of tactual and visual picture recognition with limited field of view, Perception 20 (1991) 167-177.

[60] J.M. Loomis, Tactile letter recognition under different modes of stimulus presentation, Percept. Psychophys. 16 (1974) 401-418.

[61] J.C. Bliss, J.G. Linvill, A direct translation reading aid: reading alphabetic shapes tactually, International Conference on Sensory Devices for the Blind, 1966, pp. 389-407.

[62] C.E. Kops, E.P. Gardner, Discrimination of simulated texture patterns on the human hand, Percept. Psychophys. 76 (2) (1996) 1145-1165.

[63] L.H. Boyd, W.L. Boyd, G.C. Vanderheiden, The Graphical User Interface Crisis: Danger and Opportunity, University of Wisconsin: Trace R\&D Center, UK, 1990.

[64] L.E. Krueger, David katz's der aufbau der tastwelt (the world of touch): a synopsis. Percept. Psychophys. 7 (6) (1970) 337-341.

[65] J.J. Gibson, Observations on active touch, Psychol. Rev. 69 (1962) 477-491.
[66] S.J. Lederman, M.M. Taylor, Fingertip force surface geometry and the perception of roughness by active touch, Percept. Psychophys. 12 (5) (1972) 401-408.

[67] G. Jansson, Haptic perception of outline 2D shape: the contributions of information via the skin, the joints and the muscles, in: B. Bril, A. Ledebt, G. Dietrich, A. Roby-Brami (Eds.), Advances in Perception-Action Coupling, Editions EDK, Paris, 1998, pp. 25-30.

[68] C. Ramstein, Combining haptic and Braille technologies: design issues and pilot study, ASSETS'96, Vancouver, British Columbia, Canada, 1996, pp. 37-44.

[69] T. Maucher, J. Schemmel, K. Meier, The Heidelberg tactile Vision substitution system, International Sensory Aids Conference, Exeter, UK, 23-26 May 2000.

[70] O. Gapenne, C. Lenay, J. Stewart, H. Beriot, D. Meidine, Prosthetic device and 2D form perception: the role of increasing degrees of parallelism, Proceedings of the Conference on Assistive Technology for Vision and Hearing Impairment (CVHI'2001), Castelvecchio Pascoli, Italy, 2001.

[71] N. Sribunruangrit, C. Marque, C. Lenay, O. Gapenne, C. Vanhoutte, J. Stewart, Application of parallelism concept to access graphic information with precision for blind people, EMBEC'02 2nd European Medical and Biological Engineering Conference, Vienna, Austria, 4-8 December 2002.

[72] A. Ali Ammar, O. Gapenne, C. Lenay, J. Stewart, Effect of bimodality on the perception of $2 \mathrm{D}$ forms by means of a specific assistive technology for blind persons, CVHI'2002-EURO-ASSIST-VHI-2 Conference on Assistive Technologies for Vision and Hearing Impairment, Granada, Spain, 6-9 August 2002, pp. 45-52.

[73] P. Kammermeier, G. Schmidt, Application-specific evaluation of tactile array displays for the human fingertip, IEEE/ RSJ International Conference on Intelligent Robots and Systems (IROS), Lausanne, Switzerland, 30 September-4 October 2002, pp. 2937-2942.

[74] G. Jansson, P. Pedersen, Obtaining geographical information from a virtual map with a haptic mouse, XXII International Cartographic Conference (ICC2005), A Coruna, Spain, 11-16 July 2005.

[75] I.R. Summers, Single-channel information transfer through the skin: limitations and possibilities, International Sensory Aids Conference, Exeter, UK, 23-26 May 2000.

[76] P. Bach-y-rita, J.G. Webster, W.J. Tompkins, and T. Crabb, Sensory substitution for space gloves and space robots. Space Telerobotics Workshop, Jet Propulsion Laboratory, Pasadena, CA, 20-22 January 1987, pp. 51-57.

[77] K.B. Shimoga, A survey of perceptual feedback issues in dexterous telemanipulation. Part ii. Finger touch feedback, IEEE Virtual Reality Annual International Symposium, New York, September 1993, pp. 271-279.

[78] N.J.M. Patrick, Design, Construction and Testing of a Fingertip Tactile Display for Interaction with Virtual and Remote Environments, M.Sc. Thesis, Department of Mechanical Engineering, Massachussets Institute of Technol. Cambridge, MA, 1990.

[79] B.B. Edin, R. Howe, G. Westling, M. Cutkosky, A physiological method for relaying frictional information to a human teleoperator, IEEE Trans. System Man Cybernet. 23 (2) (1993). 
[80] S.J. Lederman, R.L. Klatzky, Sensing and displaying spatially distributed fingertip forces in haptic interfaces for teleoperator and virtual environment systems, Presence: Teleoperators and Virtual Environments 8 (1) (1999) 86-103.

[81] G. Jansson, L. Monaci, Improving haptic displays: providing differentiated information at the contact areas is more important than increasing the number of areas, World Haptics 2005, Pisa, Italy, 18-20 March 2005.

[82] M.B. Cohn, M. Lam, R.S. Fearing, Tactile feedback for teleoperation, Telemanipulator Technology SPIE 1833 (1992) 240-252.

[83] C.J. Hasser, J.M. Weisenberger, Preliminary evaluation of a shape-memory alloy tactile feedback display, Symposium on Haptic Interfaces for Virtual Environments and Teleoperator Systems, ASME Winter Annual Meeting, New Orleans, LA, 1993, pp. 73-80.

[84] R.D. Howe, The shape of things to come: pin-based tactile shape displays, Proceedings of the 4th International Conference Eurohaptics 2004, Munich, Germany, 5-7 June 2004, pp. 2-11.

[85] D.A. Kontarinis, J.S. Son, W. Peine, R.D. Howe, A tactile shape sensing and display system for teleoperated manipulation, IEEE International Conference on Robotics and Automation, 1995, pp. 641-646.

[86] R.D. Howe, A force-reflecting teleoperated hand system for the study of tactile sensing in precision manipulation, IEEE International Conference on Robotics and Automation, Nice, France, May 1992, pp. 1321-1326.

[87] C. Richard, M.R. Cutkosky, Contact force perception with an ungrounded haptic interface, ASME IMECE 6th Annual Symposium on Haptic Interfaces, Dallas, TX, 15-21 November 1997.

[88] J.C. Lee, P. Dietz, D. Leigh, W. Yerazunis, S.E. Hudson, Haptic pen: a tactile feedback stylus for touch screens, Proceedings of UIST 2004, Santa Fe, NM, USA, 2004, pp. 291-294.
[89] I. Poupyrev, S. Maruyama, Tactile interfaces for small touch screens, Proceedings of UIST 2003, Vancouver, Canada, 2003, pp. 217-220.

[90] I. Poupyrev, S. Maruyama, J. Rekimoto, Ambient touch: designing tactile interfaces for handheld devices, UIST, Paris, France, 27-30 October 2002.

[91] M. Fukumoto, S. Toshaki, Activeclick: tacile feedback for touch panels, Extended Abstracts of CHI 2001, Seattle, WA, USA, 2001, pp. 121-122.

[92] M. Akamatsu, I.S. MacKenzie, T.A. Hasbrouc, A comparison of tactile auditory, and visual feedback in a pointing task using a mouse-type device, Ergonomics 38 (1995) 816-827.

[93] R.W. Soukoreff, I.S. MacKenzie, Towards a standard for pointing device evaluation: Perspectives on 27 years of fitts' law research in hci, Int. J. Human-Comput. Stud. 61 (2004) 751-789.

[94] M. Akamatsu, I.S. MacKenzie, Movement characteristics using a mouse with tactile and force feedback, Int. J. Human-Comput. Stud. 45 (1996) 483-493.

[95] H. Vitense, J.A. Jacko, V.K. Emery, Multimodal feedback: an assessment of performance and mental workload, Ergonomics 46 (1-3) (2003) 68-87.

[96] A. Cockburn, S. Brewster, Multimodal feedback for the acquisition of small targets, Ergonomics 48 (9) (2005) 1129-1150.

[97] Brewster, A.J. King, An investigation into the use of tactons to present progress information, Proceedings of IFIP Interact 2005, Rome, Italy, 2005.

[98] B. Riedel, A.M. Burton, Perception of gradient in haptic graphs: a comparison of virtual and physical stimuli, Eurohaptics, Birmingham, UK, 2001, pp. 90-92.

[99] E. Gentaz, Y. Hatwell, Role of gravitational cues in the haptic perception of orientation, Percept. Psychophys. 58 (8) (1996) 1278-1292.

[100] E. Gentaz, Y. Hatwell, The haptic oblique effect in the perception of rod orientation by blind adults. Percept. Psychophys. 60 (1) (1998) 157-167. 